\title{
Sparse Multi-Modal Topical Coding for Image Annotation
}

\author{
Lingyun Song ${ }^{\mathrm{a}}$, Minnan Luo ${ }^{\mathrm{a}, *}$, Jun Liu ${ }^{\mathrm{a}}$, Lingling Zhang ${ }^{\mathrm{a}}$, Buyue Qian ${ }^{\mathrm{a}}$, Max \\ Haifei Li ${ }^{\mathrm{b}}$, Qinghua Zheng ${ }^{\mathrm{a}}$ \\ ${ }^{a}$ MOEKLINNS Lab Department of Computer Science and Technology, Xian Jiaotong University \\ Shaanxi, 710049, China \\ ${ }^{b}$ Department of Computer Science, Union University, Jackson, TN 38305 USA
}

\begin{abstract}
Image annotation plays a significant role in large scale image understanding, indexing and retrieval. The Probability Topic Models (PTMs) attempt to address this issue by learning latent representations of input samples, and have been shown to be effective by existing studies. Though useful, PTM has some limitations in interpreting the latent representations of images and texts, which if addressed would broaden its applicability. In this paper, we introduce sparsity to PTM to improve the interpretability of the inferred latent representations. Extending the Sparse Topical Coding that originally designed for unimodal documents learning, we propose a non-probabilistic formulation of PTM for automatic image annotation, namely Sparse Multi-Modal Topical Coding. Beyond controlling the sparsity, our model can capture more compact correlations between words and image regions. Empirical results on some benchmark datasets show that our model achieves better
\end{abstract}

\footnotetext{
${ }^{*}$ Corresponding author

Email addresses: lingyun.a. song@gmail . com (Lingyun Song), minnluo@mail.xjtu.edu. cn (Minnan Luo), liukeen@mail.xjtu.edu.cn (Jun Liu), linglingzhang0929@163. com (Lingling Zhang), qianbuyue@mail.xjtu.edu.cn (Buyue Qian), hli@uu.edu (Max Haifei Li), qhzheng@mail.xjtu.edu.cn (Qinghua Zheng)
} 
performance on automatic image annotation and text-based image retrieval over the baseline models.

Keywords: Topic models, Sparse latent representation, Image annotation, Image retrieval

\section{Introduction}

The flourish development of the Internet technology has offered us numerous web images, which in turns makes the effective image retrieval more challenging. Approaches of image retrieval can be classified into two categories: Content Based Image Retrieval $[1,2]$ (CBIR) and Text Based Image Retrieval (TBIR) $[3,4]$. CBIR approaches rely on the low-level visual features that can be directly extracted from images, such as color, shape and texture. However, the performance of CBIR approaches is significantly limited by the gap between the low-level visual features and the semantic meaning of images. This is why major multimedia search engines still make use of simple TBIR approaches to perform semantic image retrieval. On the other side, TBIR approach suffers from its own limitations, e.g., it would fail if the caption texts are missing or irrelevant to the content of images. Researchers attempted to alleviate this dilemma by manually labeling images, but this would be unaffordable in terms of both time and human expenses when dealing with large scale problems. Therefore, the automatic image annotation becomes increasingly important for image retrieval tasks.

Lately, many efforts have been devoted to assigning words to unannotated images automatically. One group of researchers views image annotation as a classification problem $[5,6,7,8,9,10]$. Another group of works $[11,12,13,14,15]$ based on Probability Topic Models (PTMs) focuses on finding the conditional 
relationships between latent variable representations of sets of image regions and sets of text words. Though achieving superior performance in image annotation, these PTMs based works have two limitations:

1) Due to normalization constraints in the probabilistic formulation, existing PTMs cannot effectively control the sparsity of latent representations [16, 17]. Sparsity not only can lead to compact and high-fidelity representations, but also can facilitate the extraction of the semantic information from images [18]. It has been applied to many computer vision tasks and brought about superior performance, such as image representation [19], image classification [20, 21], image annotation $[22,23]$ and face recognition $[24,25]$.

2) PTMs such as correspondence LDA (cLDA) [11] and topic-regression multimodal Latent Dirichlet Allocation (tr-mmLDA) [12] have limitations in learning compact correlations between image regions and words. It implies that these methods are not applicable to scenarios in which each word is related to a few image regions. As shown in Fig. 1, cLDA associates the word archery with a unique image region and tr-mmLDA correlates it with all the image regions indistinguishably. In fact, archery depicts a sport which has an intimate relationship with man, archery targets and bow, whilst it is irrelevant to grass and tree.

\subsection{Overview of our model}

In order to solve the problems, we extend Sparse Topical Coding (STC) [17] and propose Sparse Multi-Modal Topical Coding (SMMTC), a novel non-probabilistic formulation of PTM for image annotation, which can be treated as a combination 


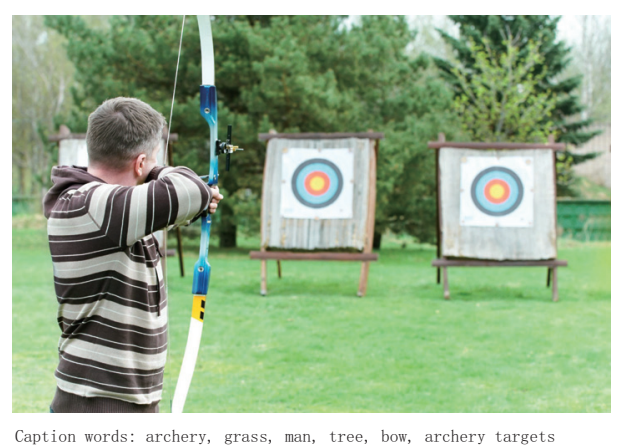

Figure 1: An illustration of compact correlations between image regions and words.

of PTM and STC. With the bag-of-word based features of multi-modal documents, SMMTC aims to learn sparse latent representations of image words, multi-modal documents and correlation codes, where the correlation codes describe the relationships between image words and caption words. Specifically, according to the generative process of a multi-modal document, a joint distribution is first defined. Then with the joint distribution, SMMTC is formulated as a loss minimization with sparsity-inducing $l_{1}$-norm regularizers. Finally, the optimization problem of SMMTC is solved by an efficient coordinate descent algorithm.

We conduct experiments on some well-known benchmark datasets to evaluate the performance of SMMTC on image annotation and text-based image retrieval tasks. The empirical results show that SMMTC outperforms the probabilistic baseline models.

\subsection{Contributions and organization}

The main contributions of this study can be summarised as follows:

1) We extend STC and propose a novel non-probabilistic formulation of PTM for image annotation. The proposed model has two appealing properties: i) It can 
effectively control the sparsity of latent representations inferred from multimodal documents. ii) It can capture more compact correlations between image regions and corresponding caption words, which avoids a word relating with a unique image region or all the image regions indistinguishably.

2) We propose an efficient coordinate descent algorithm to solve the optimization problem of the proposed model, which has closed-form solutions for updating multi-modal document codes and correlation codes.

The rest of this paper is organized as follows: Section 2 discusses the related studies. In Section 3, according to the probability generative process, we formulate SMMTC as a regularized loss minimization problem and propose an efficient coordinate descent algorithm to solve it. Section 4 presents empirical results on the tasks of image annotation and text-based image retrieval. Section 5 concludes this paper and presents future work.

\section{Related work}

Previous work on image annotation can be roughly divided into two groups. One group considers a discriminative approach. Another group of works is based on generative models.

\subsection{Discriminative approaches}

These discriminative approaches view image annotation as a classification problem. Each caption word is regarded as a class and the corresponding classifier is separately trained to predict whether the test image belongs to the class. For example, Li and Wang [6] presented an automatic linguistic indexing of pictures (ALIP) system, in which categorized images are used to train a set of models each 
corresponding to a concept. Carneiro et al. [26] proposed a supervised multiclass labeling (SML) framework, which establishes a one-to-one correspondence between semantic labels and image groups. Tao et al. [27] presented a new Hessian Regularized Support Vector Machine (HRSVM) for mobile image annotation on the cloud, in which the Hessian regularization is constructed by using a large number of unlabeled images.

Some other researchers assumed that an image containing multiple objects should be annotated with a set of labels. For this reason, multi-label learning is introduced to assign multiple labels for each image [28, 29, 30, 31]. For example, $\mathrm{Xu}$ et al. [28] proposed a multi-label learning algorithm for predicting image annotations, which concentrates on the tail singular values of the multi-label predictor. Wei et al. [31] introduced the nearest neighbor data editing technique to multi-label image classification. Luo et al. [30] proposed a manifold regularized multi-task learning (MRMTL) algorithm for multi-label image classification.

Another group of researchers applied multi-view learning [9, 32, 33] algorithms to image annotation, which generate multiple views of images based on various kinds of image features, such as color, shape and texture. For example, Liu and Tao [34] presented a multi-view Hessian Regularization (mHR) framework for image annotation. In mHR, multi-view features and Hessian regularizations are combined to explore the complementary properties of different features. $\mathrm{Xu}$ et al. [33] proposed a large-margin multi-view information bottleneck (LMIB) algorithm for image annotation. Luo et al. [35] extended Matrix completion (MC) to a multi-view Matrix completion (MVMC) framework for multi-label classification, which combines the MC outputs of different views. Xu et al. [32] integrated the complementary information in multiple views to discover the latent 
intact representations for image annotation. Luo et al. [36] presented a multi-view vector-valued manifold regularization (MV3MR) framework, which introduces multi-kernel learning (MKL) to the vector-valued function and can handle the multi-view features of images.

However, most discriminative methods take an image as a whole and neglect the fact that one caption word is generally related to a few image regions rather than the entire image. In contrast, the proposed algorithm can capture compact correlations between image regions and corresponding caption words, where a word is correlated with a few image regions.

\subsection{Generative model based approaches}

This group of approaches attempts to estimate the joint probability of image and keywords. Jeon et al. [37] proposed the Cross-media Relevance Models (CMRM) to estimate the joint probability of visual-based keywords and text-based semantic keywords. CMRM is subsequently improved by Continuous-space Relevance Model (CRM) [38], which can directly model continuous features. Wang et al. [39] proposed a content-based image annotation refinement (CIAR) algorithm, which formulates image annotation refinement process as a Markov process and defines the candidate annotations as the states of a Markov chain.

In recent years, a group of works based on PTMs has received significant attention. One representative work is presented by Blei and Jordan [11]. They extended LDA and proposed cLDA to model the joint distribution of images and words as well as the conditional relationships between them. Furthermore, Wang and Blei et al. [15] proposed a multi-class supervised LDA model, which uses the same generative process as cLDA to model annotations. In the generative process of cLDA, all of image region descriptions are first generated from an 
LDA model. Then, to guarantee latent variables used in generating the caption words are those that generate the image regions, each caption word directly shares a latent variable with a randomly selected image region. Therefore, cLDA and multi-class sLDA associate each word with a unique image region, which are not applicable to scenarios that one word describe more than one image regions.

Putthividhya et al. [12] considered that the severe constraint on associations between image regions and words in cLDA is ill-advised. For example, sometimes a word describes an image from an overall perspective. They extended cLDA and proposed tr-mmLDA to solve this issue. In tr-mmLDA, the latent variables of each modality are learned separately and a regression module is constructed to regulate the correlations between them. Thus the number of latent variables of images can be different from that of words. In this way, tr-mmLDA relaxes the constraint and allows a word to be correlated with all image regions instead of only one region. Nevertheless, it is not always appropriate to associate one word with all image regions indiscriminately. As seen in Fig. 1, the word archery only has relationships with several image regions.

Though PTMs focus on learning latent semantics of complex data and have shown promising performance in many computer vision tasks, they have a limitation in effectively controlling the sparsity of the inferred representations [17]. Sparsity is crucial in learning meaningful and interpretable latent representations of images [18]. Though the sparse prior (e.g., Dirichlet[40]) or auxiliary variables [41] used in PTM can indirectly bring about a sparsity bias over the posterior representations, it has been proved that directly imposing posterior regularization is a better approach for achieving sparsity [16]. However, due to normalization constraints in PTMs, it is useless to directly impose a sparsity inducing regu- 
larizer [42, 43]. In contrast, by relaxing the normalization constraints, some non-probabilistic models $[17,44]$ can effectively control the sparsity of inferred representations through directly using appropriate regularizers.

As a non-probabilistic formulation of topic models without normalization constraints, STC not only can discover the latent semantic structures of data corpora, but also can effectively control the sparsity of inferred representations by directly using a sparsity-inducing $l_{1}$-regularizer. However, STC is originally designed for modeling text documents, which can't work on multi-modal documents that consist of images and texts. In this paper, we extend STC and propose SMMTC for image annotation, which can effectively control the sparsity of inferred representations and find compact correlations between image regions and words. Experimental results show that the proposed algorithm achieves superior image annotation performance.

\section{Sparse multi-modal topical coding}

In this section, we propose a SMMTC model for image annotation, which extends cLDA under the non-probabilistic formulation [17]. In other words, SMMTC is a combination of CLDA and STC. In order to facilitate understanding, we first introduce a probabilistic generative process. Second, according to the joint distribution described in 3.2, we cast SMMTC as finding a Maximum-a-Posteriori (MAP) estimate. Finally, we propose an efficient coordinate descent algorithm to solve the optimization problem of SMMTC.

\subsection{A probabilistic generative process for SMMTC}

SMMTC adopts bag-of-word based features for both images and caption texts. Let $B \subseteq \mathbb{N}_{+}$be a index set of image words and $|B|=M$. Let $V \subseteq \mathbb{N}_{+}$be a index 
set of caption words and $|V|=N$.

An image and the corresponding caption text are treated as a multi-modal document. We denote a corpus of $D$ multi-modal documents by $\mathscr{M}=\left\{\left(R^{d}, W^{d}\right)\right\}_{d=1}^{D}$, where $\left(R^{d}, W^{d}\right)$ represents the $d$-th multi-modal document. $R^{d}=\left\{r_{m}^{d}: m \in I^{d}\right\}$ represents the image, where $r_{m}^{d}$ is the count of the image word $m$ and $I^{d} \subset B$ is the index set of image words that appear in the $d$-th document. $W^{d}=\left\{w_{n}^{d}: n \in J^{d}\right\}$ represents the caption text, where $w_{n}^{d}$ is the count of the caption word $n$ and $J^{d} \subset V$ is the index set of caption words that appear in the $d$-th document. In a multimodal document, different image words may have the same count number, so do different caption words. Therefore, $R^{d}$ and $W^{d}$ are defined as multisets [45] which can contain repeated elements.

With $K$ topical bases, the image dictionary and the text dictionary are denoted by $\Psi=\left(\psi_{i j}\right) \in \mathbb{R}_{+}^{K \times M}$ and $\Phi=\left(\phi_{i j}\right) \in \mathbb{R}_{+}^{K \times N}$, respectively. The $k$-th row of $\Psi$ is denoted by $\Psi_{k} \in \mathscr{P}^{M}(k=1,2, \cdots, K)$, where $\mathscr{P}^{M}$ represents a $(M-1)$-simplex; the $k$-th row of $\Phi$ is denoted by $\Phi_{k}$. $\in \mathscr{P}^{N}$, where $\mathscr{P}^{N}$ represents a $(N-1)$ simplex. $\Psi_{k}$. and $\Phi_{k}$. are normalized distributional vectors over image words and caption words, respectively. $\Psi_{\cdot m}$ and $\Phi_{\cdot n}$ are used to denote the $m$-th column of $\Psi$ and the $n$-th column of $\Phi$, respectively. SMMTC aims to project the input image words and caption words into a high-level representation semantic space, where image words and caption words are represented as unnormalized distributional vectors over $K$ topical bases.

The graphical model representation of SMMTC is shown in Fig. 2. We first sample the image dictionary $\Psi$ and the text dictionary $\Phi$ from uniform distributions on $\mathscr{P}^{M}$ and $\mathscr{P}^{N}$, respectively. Then, in the generative process of $d$-th multi-modal document, image words are first generated and subsequently 


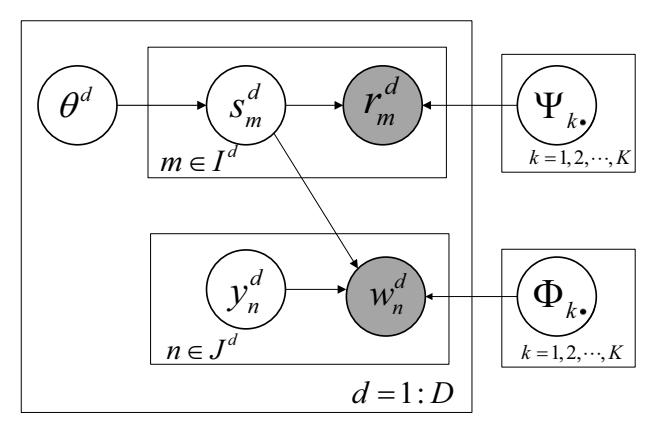

Figure 2: Graphical model representation of SMMTC.

caption words are generated. Following [17], we assume that each image word code $\mathbf{s}_{m}^{d} \in \mathbb{R}_{+}^{K}\left(m \in I^{d}\right)$ is conditionally independent given the document code $\theta^{d} \in \mathbb{R}_{+}^{K}$ and each image word count $r_{m}^{d}$ is sampled from a distribution whose mean parameter is $\left(s_{m}^{d}\right)^{\top} \Psi_{\cdot m}$. For the caption word $n$, we draw the correlation code $\mathbf{y}_{n}^{d} \in \mathbb{R}_{+}^{\left|I^{d}\right|}\left(n \in J^{d}\right)$, which allows the caption word code $\mathbf{z}_{n}^{d} \in \mathbb{R}_{+}^{K}$ to be predicted from a linear combination of image word codes, i.e., $\mathbf{z}_{n}^{d}=S^{d} \mathbf{y}_{n}^{d}$, where $S^{d}=\left[\mathbf{s}_{1}^{d}, \mathbf{s}_{2}^{d}, \cdots, \mathbf{s}_{\left|I^{d}\right|}^{d}\right]^{1} \in \mathbb{R}_{+}^{K \times\left|I^{d}\right|}$. We also assume that the caption word count $w_{n}^{d}$ follows a distribution whose mean parameter is $\left(\mathbf{z}_{n}^{d}\right)^{\top} \Phi_{. n}$. Formally, each multimodal document can be described in the following process.

For the $d$-th multi-modal document $\left(R^{d}, W^{d}\right)$ :

1 Draw a multi-modal document code $\theta^{d}$ from $p\left(\theta^{d}\right)$.

2 For each observed image word $m \in I^{d}$ :

(a) Draw the image word code $\mathbf{s}_{m}^{d}$ from $p\left(\mathbf{s}_{m}^{d} \mid \theta^{d}\right)$.

(b) Draw the observed image word count $r_{m}^{d}$ from $p\left(r_{m}^{d} \mid\left(\mathbf{s}_{m}^{d}\right)^{\top} \Psi_{\cdot m}\right)$.

3 For each observed caption word $n \in J^{d}$ :

\footnotetext{
${ }^{1} s_{i}^{d}$ represents the $i$-th image word code in the $d$-th multi-modal document $\left(i=1,2, \cdots,\left|I^{d}\right|\right)$.
} 
(a) Draw the correlation code $\mathbf{y}_{n}^{d}$ from $p\left(\mathbf{y}_{n}^{d}\right)$. Then compute caption word code by $\mathbf{z}_{n}^{d}=S^{d} \mathbf{y}_{n}^{d}$.

(b) Draw the observed caption word count $w_{n}^{d}$ from $p\left(w_{n}^{d} \mid\left(\mathbf{z}_{n}^{d}\right)^{\top} \Phi_{\cdot n}\right)$.

We will discuss the choice of $p\left(\theta^{d}\right), p\left(\mathbf{s}_{m}^{d} \mid \theta^{d}\right), p\left(r_{m}^{d} \mid\left(\mathbf{s}_{m}^{d}\right)^{\top} \Psi \cdot m\right), p\left(\mathbf{y}_{n}^{d}\right)$ and $p\left(w_{n}^{d} \mid\left(\mathbf{z}_{n}^{d}\right)^{\top} \Phi_{. n}\right)$ in Section 3.2. It is noteworthy that a new latent variable, namely correlation code, is introduced in SMMTC to capture compact correlations between image words and caption words. The sparsity of correlation codes can be directly controlled via imposing a sparsity-inducing $l_{1}$-norm regularizer. It indicates that SMMTC correlates each caption word with a few image words.

\subsection{MAP estimate of SMMTC}

According to the generation procedure described above, a joint distribution on the latent variables in a multi-modal document is defined as follows:

$$
\begin{aligned}
& p\left(\theta^{d}, R^{d}, W^{d}, S^{d}, Y^{d} \mid \Phi, \Psi\right) \\
= & p\left(\theta^{d}\right) \prod_{m \in I^{d}} p\left(\mathbf{s}_{m}^{d} \mid \theta^{d}\right) p\left(r_{m}^{d} \mid \mathbf{s}_{m}^{d}, \Psi\right) \cdot \prod_{n \in J^{d}} p\left(\mathbf{y}_{n}^{d}\right) p\left(w_{n}^{d} \mid S^{d}, \mathbf{y}_{n}^{d}, \Phi\right),
\end{aligned}
$$

where $Y^{d}=\left[\mathbf{y}_{1}^{d}, \mathbf{y}_{2}^{d}, \cdots, \mathbf{y}_{\left|J^{d}\right|}^{d}\right] \in \mathbb{R}_{+}^{\left|I^{d}\right| \times\left|J^{d}\right|}$ represents correlation codes for caption words in the $d$-th multi-modal document. In the framework of SMMTC, Laplace prior $p\left(\theta^{d}\right) \propto \exp \left(-\lambda\left\|\theta^{d}\right\|_{1}\right)$ and $p\left(\mathbf{y}_{n}^{d}\right) \propto \exp \left(-\mu\left\|\mathbf{y}_{n}^{d}\right\|_{1}\right)$ are chosen to achieve sparse $\theta^{d}$ and $\mathbf{y}_{n}^{d}$. We define $p\left(\mathbf{s}_{m}^{d} \mid \theta^{d}\right)$ with a composite distribution

$$
p\left(\mathbf{s}_{m}^{d} \mid \theta^{d}\right) \propto \exp \left(-\gamma\left\|\mathbf{s}_{m}^{d}-\theta^{d}\right\|_{2}^{2}-\rho\left\|\mathbf{s}_{m}^{d}\right\|_{1}\right)
$$

which is super-Gaussian [46]. The $l_{1}$-norm is used to find sparse image word codes, which are limited close to the corresponding multi-modal document code 
by a normal regularizer. The hyper-parameters $(\lambda, \mu, \gamma, \rho)$ are non-negative and predefined via cross-validation.

In order to specify the joint distribution, we also need to define the image word likelihood model $p\left(r_{m}^{d} \mid \mathbf{s}_{m}^{d}, \Psi\right)$ and the caption word likelihood model $p\left(w_{n}^{d} \mid S^{d}, \mathbf{y}_{n}^{d}, \Phi\right)$. As image word counts are discrete, we follow [17] and use the Poisson distribution to generate the observations, i.e.,

$$
p\left(r_{m}^{d} \mid \mathbf{s}_{m}^{d}, \Psi\right)=\operatorname{Poiss}\left(r_{m}^{d} ;\left(\mathbf{s}_{m}^{d}\right)^{\top} \Psi_{\cdot m}\right)
$$

where Poiss $(x ; v)=v^{x} e^{-v} / x$ !. We also use the Poisson distribution to generate the observation of each caption word count, i.e., $p\left(w_{n}^{d} \mid S^{d}, \mathbf{y}_{n}^{d}, \Phi\right)=\operatorname{Poiss}\left(w_{n}^{d} ;\left(\mathbf{z}_{n}^{d}\right)^{\top} \Phi_{\cdot n}\right)$, where $\mathbf{z}_{n}^{d}=S^{d} \mathbf{y}_{n}^{d}$. Setting the linear combination $\left(s_{m}^{d}\right)^{\top} \Psi_{\cdot m}$ and $\left(\mathbf{z}_{n}^{d}\right)^{\top} \Phi_{\cdot n}$ as mean parameters of the Poisson distributions, we can conveniently constrain the feasible domains of image word codes and caption word codes for good interpretation via restricting $s_{m}^{d}$ and $y_{n}^{d}$ to non-negative codes [44].

Let $\Gamma=\{\Psi, \Phi\}$ be the dictionary for corpus $\mathscr{M}$ and $\Delta=\{\Theta, S, Y\}$ denote the codes for all the multi-modal documents in $\mathscr{M}$. Specifically, $\Theta=\left\{\theta^{d}: d=1, \cdots, D\right\}$ represents all the multi-modal documents codes, $S=\left\{\mathbf{s}_{m}^{d}: m \in I^{d}, d=1, \cdots, D\right\}$ represents all the image word codes and $Y=\left\{\mathbf{y}_{n}^{d}: n \in J^{d}, d=1, \cdots, D\right\}$ represents all the correlation codes. Let $R=\left\{R^{d}: d=1, \cdots, D\right\}$ and $W=\left\{W^{d}: d=\right.$ $1, \cdots, D\}$ denote all the images and all the caption text in $\mathscr{M}$, respectively.

With the joint distribution (1), SMMTC is defined as finding a MAP estimate with dictionary learning, which can be formulated as solving the following opti- 
mization problem:

$$
\begin{array}{ll}
\min _{\Delta, \Gamma} & l(S, \Psi ; R)+h(S, \Phi, Y ; W)+\Omega(\Theta, S, Y) \\
\text { s.t. } & \theta^{d} \geq 0, \forall d ; \mathbf{s}_{m}^{d} \geq 0, \forall d, \forall m \in I^{d} ; \mathbf{y}_{n}^{d} \geq 0, \forall d, \forall n \in J^{d} ; \\
& \Psi_{k \cdot} \in \mathscr{P}^{M} ; \Phi_{k} \in \mathscr{P}^{N}, \forall k,
\end{array}
$$

where the objective function is the negative logarithm of the posterior $p(\Theta, S, Y, \Psi$ $, \Phi \mid W, R)$ with a constant omitted; $l(S, \Psi ; R)=-\sum_{d} \sum_{m \in I^{d}} \log \operatorname{Poiss}\left(r_{m}^{d} ;\left(\mathbf{s}_{m}^{d}\right)^{\top} \Psi \cdot m\right)$ represents the negative log-likelihood of image word counts and $h(S, \Phi, Y ; W)=$ $-\sum_{d} \sum_{n \in J^{d}} \log$ Poiss $\left(w_{n}^{d} ;\left(S^{d} \mathbf{y}_{n}^{d}\right)^{\top} \Phi_{\cdot n}\right)$ represents the negative log-likelihood of caption word counts. $\left(s_{m}^{d}\right)^{\top} \Psi_{\cdot m}$ is the reconstruction of the image word count $r_{m}^{d}$ and $\left(S^{d} \mathbf{y}_{n}^{d}\right)^{\top} \Phi_{\cdot n}$ is the reconstruction of the caption word count $w_{n}^{d}$. The regularization term is formulated as

$$
\Omega(\Theta, S, Y)=\lambda \sum_{d}\left\|\theta^{d}\right\|_{1}+\sum_{d} \sum_{m \in I^{d}}\left(\gamma\left\|\mathbf{s}_{m}^{d}-\theta^{d}\right\|_{2}^{2}+\rho\left\|\mathbf{s}_{m}^{d}\right\|_{1}\right)+\mu \sum_{d} \sum_{n \in J^{d}}\left\|\mathbf{y}_{n}^{d}\right\|_{1} .
$$

$\theta^{d}, \mathbf{s}_{m}^{d}$ and $\mathbf{y}_{n}^{d}$ in optimization problem (3) are constrained to be non-negative for two reasons: i) Image word counts and caption word counts are non-negative. ii) Non-negative constraints can potentially result in sparser and more interpretable representations [44]. Each non-negative element of $\theta^{d}$ can be interpreted as the correlation between a topic and the $d$-th document. Each non-negative element of $\mathbf{s}_{m}^{d}$ can be interpreted as the correlation between a topic and the $m$-th image word in the d-th document. 


\subsection{Optimization algorithm}

As the optimization problem (3) is bi-convexity over the multi-modal document dictionary $\Gamma$ and the code collection $\Delta$ for all the multi-modal documents in $\mathscr{M}$, a coordinate descent algorithm is naturally used to solve it. This algorithm is typically used in sparse coding [17, 47, 42]. Specifically, the update of multi-modal document codes $\Theta$, image word codes $S$ and correlation codes $Y$ are alternatively performed given the fixed $\Gamma$; then the image dictionary $\Psi$ and the text dictionary $\Phi$ are updated when $\Delta$ is fixed. We summarize the coordinate descent algorithm for SMMTC in Algorithm 1.

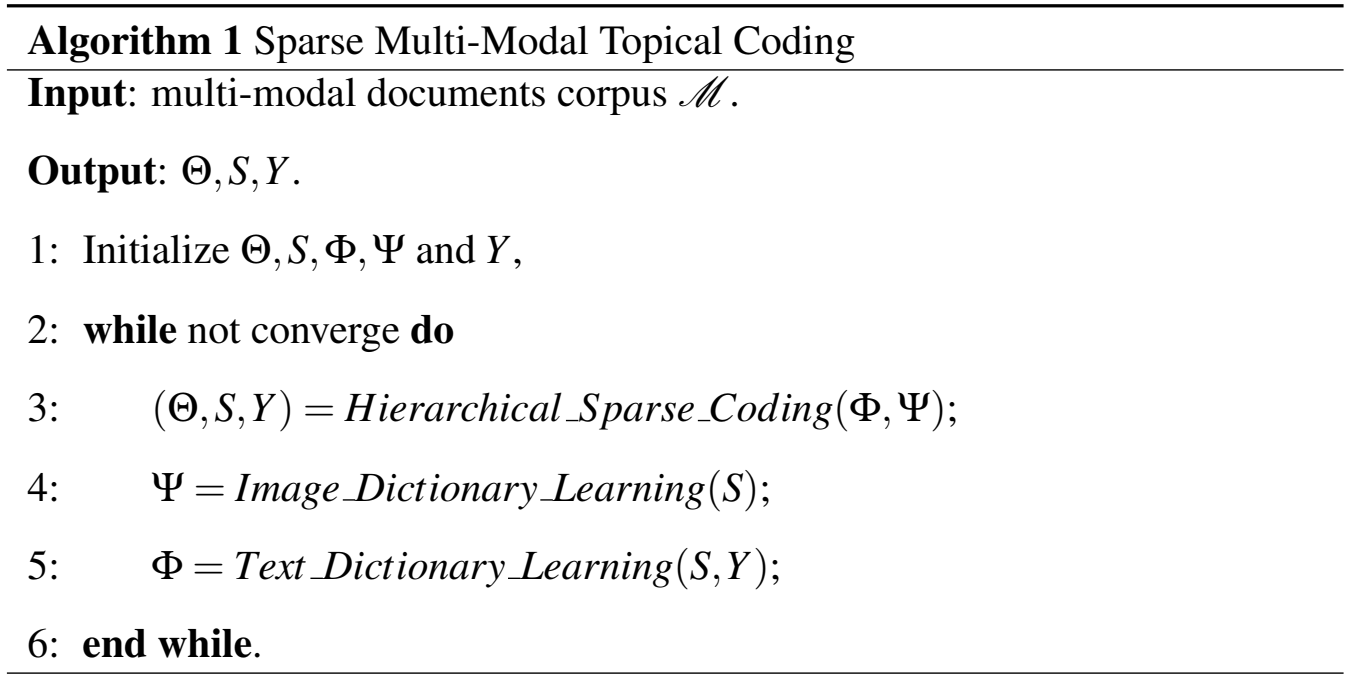

\subsubsection{Hierarchical sparse coding for multi-modal documents}

In this step, image word codes, multi-modal document codes and correlation codes are solved with the fixed dictionaries of images and text. Because of the conditional independency, we can perform this step for each multi-modal docu- 
ment separately by solving the optimization problem

$$
\begin{aligned}
& \min _{\theta^{d}, s_{m}^{d}, y_{n}^{d}} \sum_{m \in I^{d}} l\left(\mathbf{s}_{m}^{d}, \Psi\right)+\sum_{n \in J^{d}} h\left(S^{d}, \mathbf{y}_{n}^{d}, \Phi\right)+\Omega^{d}\left(\theta^{d}, \mathbf{s}_{m}^{d}, \mathbf{y}_{n}^{d}\right) \\
& \text { s.t. } \quad \theta^{d} \geq 0 ; \mathbf{s}_{m}^{d} \geq 0, \forall m \in I^{d} ; \mathbf{y}_{n}^{d} \geq 0, \forall n \in J^{d},
\end{aligned}
$$

where the regularization term with respect to the $d$-th multi-modal document is formulated as

$$
\Omega^{d}\left(\theta^{d}, \mathbf{s}_{m}^{d}, \mathbf{y}_{n}^{d}\right)=\lambda\left\|\theta^{d}\right\|_{1}+\sum_{m \in I^{d}}\left(\gamma\left\|\mathbf{s}_{m}^{d}-\theta^{d}\right\|_{2}^{2}+\rho\left\|\mathbf{s}_{m}^{d}\right\|_{1}\right)+\mu \sum_{n \in J^{d}}\left\|\mathbf{y}_{n}^{d}\right\|_{1}
$$

With a coordinate descent method, the optimization problem (4) can be alternatively solved by addressing the following three optimization problems.

(1) Optimization over $\mathbf{s}_{m}^{d}$ : when $\theta^{d}$ and $\mathbf{y}_{n}^{d}$ are fixed, each $\mathbf{s}_{m}^{d}$ can be obtained by solving the optimization problem

$$
\begin{aligned}
& \min _{\mathbf{s}_{m}^{d}} \mathscr{L}\left(\mathbf{s}_{m}^{d}\right) \\
& \text { s.t. } \quad \mathbf{s}_{m}^{d} \geq 0, \forall m \in I^{d},
\end{aligned}
$$

where objective function $\mathscr{L}\left(\mathbf{s}_{m}^{d}\right)=l\left(\mathbf{s}_{m}^{d}, \Psi\right)+\sum_{n \in J^{d}} h\left(S^{d}, \mathbf{y}_{n}^{d}, \Phi\right)+\gamma\left\|\mathbf{s}_{m}^{d}-\theta^{d}\right\|_{2}^{2}$ $+\rho\left\|\mathbf{s}_{m}^{d}\right\|_{1}$. For the convexity of objective function above, a projected gradient descent algorithm is naturally utilized to solve the optimization problem (5). Therefore, $\mathbf{s}_{m}^{d}$ is updated at each iteration by

$$
\left[\mathbf{s}_{m}^{d}\right]^{\text {new }}=P\left(\left[\mathbf{s}_{m}^{d}\right]^{\text {old }}-t \nabla_{\mathbf{s}_{m}^{d}} \mathscr{L}\right),
$$


where $t$ is the step size; $P$ is a projection operator regarding to positive half space of $\mathbb{R}^{K}$; and the gradient of $\mathscr{L}$ with respect to $\mathbf{s}_{m}^{d}$ is computed as

$$
\begin{aligned}
\nabla_{\mathbf{s}_{m}^{d}} \mathscr{L} & =\frac{\partial \mathscr{L}}{\partial \mathbf{s}_{m}^{d}}=\frac{\partial l\left(\mathbf{s}_{m}^{d}, \Psi\right)}{\partial \mathbf{s}_{m}^{d}}+\frac{\partial \sum_{n \in J^{d}} h\left(S^{d}, \mathbf{y}_{n}^{d}, \Phi\right)}{\partial \mathbf{s}_{m}^{d}}+2 \gamma\left(\mathbf{s}_{m}^{d}-\theta^{d}\right)+\rho \\
& =\left(1-\frac{r_{m}^{d}}{\left(\mathbf{s}_{m}^{d}\right)^{\top} \Psi_{\cdot m}}\right) \Psi_{\cdot m}+\sum_{n \in J^{d}}\left(1-\frac{w_{n}^{d}}{\left(\mathbf{z}_{n}^{d}\right)^{\top} \Phi_{\cdot n}}\right) y_{n m}^{d} \Phi_{\cdot n}+2 \gamma\left(\mathbf{s}_{m}^{d}-\theta^{d}\right)+\rho
\end{aligned}
$$

where $\mathbf{z}_{n}^{d}=S^{d} \mathbf{y}_{n}^{d}$.

When updating each image word, the complexity of the gradient computation is $O(K b)$, in which $b=\max _{d \in\{1,2, \cdots, D\}}\left|J^{d}\right|$. As there are $\left|I^{d}\right|$ image words in the $d$-th multi-modal document, the complexity of computing all the image word codes is $O(K a b)$, where $a=\max _{d \in\{1,2, \cdots, D\}}\left|I^{d}\right|$.

(2) Optimization over $\theta^{d}$ : when $S^{d}$ is fixed, $\theta^{d}$ can be obtained by solving convex optimization problem

$$
\begin{aligned}
& \min _{\theta^{d}} \lambda\left\|\theta^{d}\right\|_{1}+\gamma \sum_{m \in I^{d}}\left\|\mathbf{s}_{m}^{d}-\theta^{d}\right\|_{2}^{2} \\
& \text { s.t. } \quad \theta^{d} \geq 0 .
\end{aligned}
$$

According to the proposition 1 in [17], the closed-form solution is

$$
\theta_{k}^{d}=\max \left(0, \bar{s}_{k}^{d}-\frac{\lambda}{2 \gamma\left|I^{d}\right|}\right)
$$

where $\bar{s}_{k}^{d}=\left(1 /\left|I^{d}\right|\right) \sum_{m \in I^{d}} s_{m k}^{d}$ for $k=1,2, \cdots, K$. The complexity of computing $\theta^{d}$ is $O(K a)$.

(3) Optimization over $\mathbf{y}_{n}^{d}$ : after obtaining the optimal solutions of $\mathbf{s}_{m}^{d}$, we update each correlation code $\mathbf{y}_{n}^{d}$ by minimizing the log-Poisson loss of caption 
word counts with a $l_{1}$-norm regularizer, i.e.,

$$
\begin{aligned}
& \min _{\mathbf{y}_{n}^{d}} h\left(S^{d}, \mathbf{y}_{n}^{d}, \Phi\right)+\mu\left\|\mathbf{y}_{n}^{d}\right\|_{1} \\
& \text { s.t. } \quad \mathbf{y}_{n}^{d} \geq 0 .
\end{aligned}
$$

Due to the conditional independency, we can optimize the correlation code for each multi-modal document separately and omit the superscript letter $d$ for better representation. Let $g\left(\mathbf{y}_{n}\right)=h\left(S, \mathbf{y}_{n}, \Phi\right)+\mu \sum_{m} y_{n m}$. Then we compute each $y_{n m}$ alternatively. The solution is $y_{n m}=\max \left(0, v_{m}\right)$, where $v_{m}=\arg \min _{y_{n m}} g\left(\mathbf{y}_{n}\right)$ with $y_{n i}\left(i \in I^{d} \backslash\{m\}\right)$ fixed at current solutions [17]. Setting the gradient

$$
\begin{aligned}
\nabla_{y_{n m}} g & =\left(1-\frac{w_{n}}{\mathbf{y}_{n}^{\top} S^{\top} \Phi_{\cdot n}}\right) \mathbf{s}_{m}^{\top} \Phi_{\cdot n}+\mu \\
& =\left(1-\frac{w_{n}}{\sum_{i \in I^{d} \backslash\{m\}} \sum_{j=1}^{K} y_{n i} \mathbf{s}_{i j} \phi_{j n}+y_{n m} \sum_{j=1}^{K} \mathbf{s}_{m j} \phi_{j n}}\right) \mathbf{s}_{m}^{\top} \Phi_{\cdot n}+\mu=0,
\end{aligned}
$$

then, we have $y_{n m}=\max \left(0, v_{m}\right)$ with

$$
v_{m}=\left(\frac{w_{n}}{1+\frac{\mu}{\mathbf{s}_{m}^{\top} \Phi \cdot n}}-u_{1}\right) / u_{2}
$$

where $u_{1}=\sum_{i} \sum_{j} y_{n i} \mathbf{s}_{i j} \phi_{j n}$ and $u_{2}=\sum_{j} \mathbf{s}_{m j} \phi_{j n}$. The complexity of computing each correlation code $y_{n}^{d}$ is $O(K a)$. As the $d$-th multi-modal document has $\left|J^{d}\right|$ correlation codes, the complexity of computing all the correlation codes is $O(K a b)$.

\subsubsection{Dictionaries learning for images and text}

After inferring the codes $\Delta=\{\Theta, S, Y\}$ for all the multi-modal documents, the image dictionary $\Psi$ and the text dictionary $\Phi$ can be updated by minimizing 
$l(S, \Psi ; R)$ and $h(S, \Phi, Y ; W)$, respectively. The log-Poisson loss is convex with respect to the corresponding dictionary, which can be efficiently solved by a wellused projected gradient descent algorithm [48].

\subsubsection{Complexity}

As shown in Algorithm 1, the time complexity of SMMTC is determined by three parts: Hierarchical_Sparse_Coding, Image_Dictionary_Learning and Text_Di ctionary_learning.

In Hierarchical_Sparse_Coding, the image word code $s_{m}^{d}$, the multi-modal document code $\theta^{d}$ and the correlation code $y_{n}^{d}$ are alternatively computed. Therefore, the complexity of Hierarchical_Sparse_Coding over $D$ multi-modal documents is $O(D \cdot(K a b+K a+K a b))=O(D \cdot K a b)$.

The image dictionary $\Psi$ and the text dictionary $\Phi$ are updated by using a projected gradient descent algorithm. The complexity of updating $\Psi$ and $\Phi$ are $O(K a D)$ and $O(K a b D)$, respectively.

In summary, the complexity of SMMTC is $O(t \cdot(D \cdot K a b+K a b D+K a D))=$ $O(D K t a b)$, where $t$ denotes the iteration number of the while loop in Algorithm 1.

\section{Experimental evaluations}

In this section, we first describe the datasets and baseline models used in our experiments. Second, we demonstrate the characteristics of SMMTC. Finally, we present experimental results of image annotation and text-based image retrieval. Compared with the baseline models, the proposed model achieves superior performance when guaranteeing the sparsity of representations inferred from multimodal documents. 


\subsection{Datasets and baseline models}

We conduct experiments over two benchmark datasets: Corel 5K and a subset of the LabelMe [49]. Corel 5K was first used in [50]. From then on, it has become a benchmark dataset which is intensively used in image annotation and retrieval experiments [5, 12, 51, 52]. Corel 5K contains 5000 images from 50 Corel Stock Photo CDs. Each CD consists of 100 images of the same topic and each image is associated with 1-5 caption words. The size of each image is reduced to $117 \times 181$. 4500 images are used in training (90 images per CD) and 500 images are used for testing purposes (10 images per CD).

For the LabelMe dataset, we adopt the 8-category subset that used in $[12,15]$ for fair comparisons. This subset contains 2687 images from 8 outdoor scene categories including "coast," "forest," "highway," "inside city," "mountain," "open country," "street" and "tall building." The size of each image is $256 \times 256$. $80 \%$ of the images in each category are used in training and $20 \%$ of the images in each category are used in testing.

Following the work in [12], we need to preprocess the images as follows. First, we segment each training image into some $20 \times 20$ patches by sliding a window with a 20-pixel interval. Second, we extract 128-dim SIFT descriptors from the $20 \times 20$ gray-scale patches and use additional 36-dim robust color descriptors [53] to complement the SIFT descriptors. Third, we use $k$-means to quantize the combined 164-dim features into 256 clusters and each center of clusters is treated as a word in the image vocabulary. Given a new test image, we identify its image words via mapping its features to the clusters using k-NN algorithm. Considering the different statistics in two datasets, we construct two image word vocabularies separately. 
We choose two well-known PTMs as our baselines. The datasets and image features used in our experiments are consistent with the baselines.

1) CLDA: a heuristic model that extends LDA for automatic image annotation and has been extensively selected as a baseline $[12,15,14]$. It assumes that each caption word is correlated with a unique image region and finds conditional relationships between latent variable representations of image regions and caption words.

2) Tr-mmLDA: a popular topic model that generalizes cLDA and introduces a regression module to regulate the correlations between image regions and corresponding caption words. Different from cLDA, tr-mmLDA allows each caption word to be associated with more than one image regions.

\subsection{Characteristics of SMMTC}

First, we analyze the efficiency of the proposed model by observing the convergence over two benchmark datasets. Second, we show some image word codes and multi-modal document codes to illustrate the effectiveness of SMMTC on controlling the sparsity of referred latent representations. As sparsity ratio of correlation codes is specifically demonstrated in Section 4.3.2, we will not display the correlation codes here.

Fig. 3 shows the variation of objective function values of the optimization problem (3) when the number of topical bases $K$ is fixed at 100. From the figure, we can observe that the proposed algorithm converges within a few iterations, which is very efficient. The reason is that we adopt an efficient coordinate descent algorithm to solve the optimization problem, which has closed-form solutions for updating multi-modal document codes and correlation codes. 


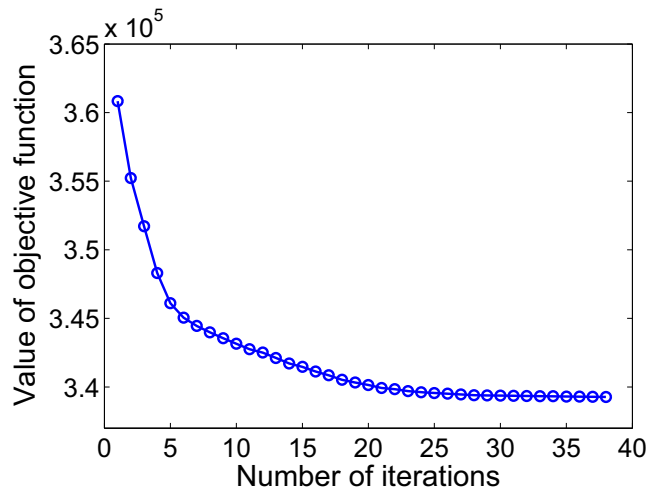

(a) Corel dataset

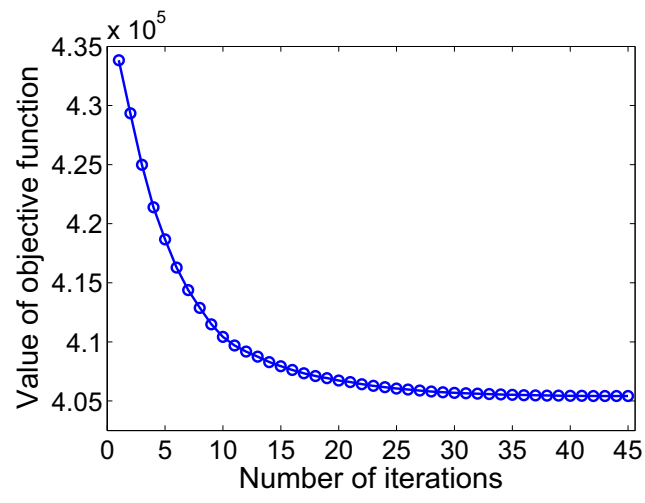

(b) LabelMe dataset

Figure 3: Convergence test on different datasets.

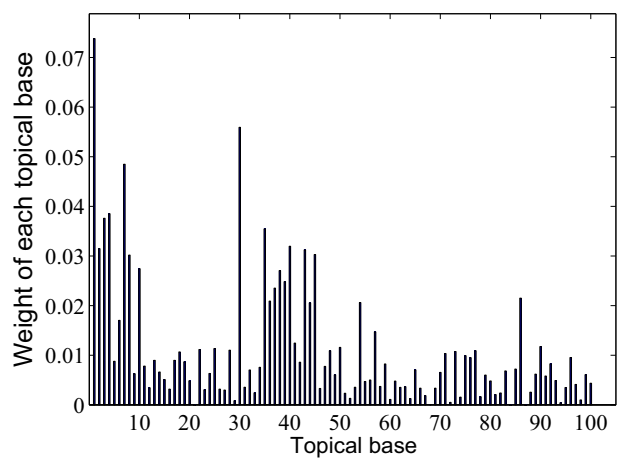

(a) Average document code of opencountry

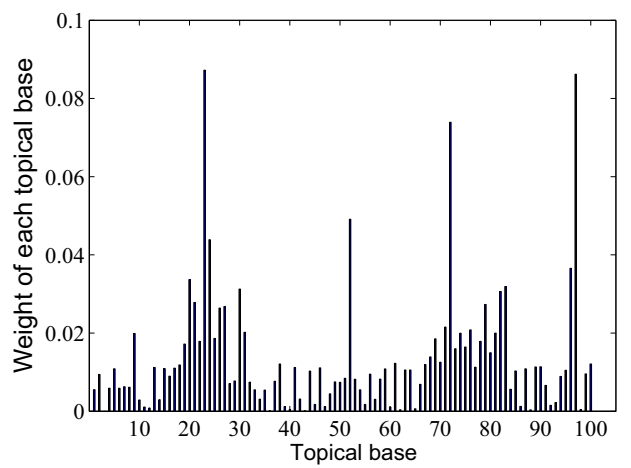

(c) Average document code of tallbuilding

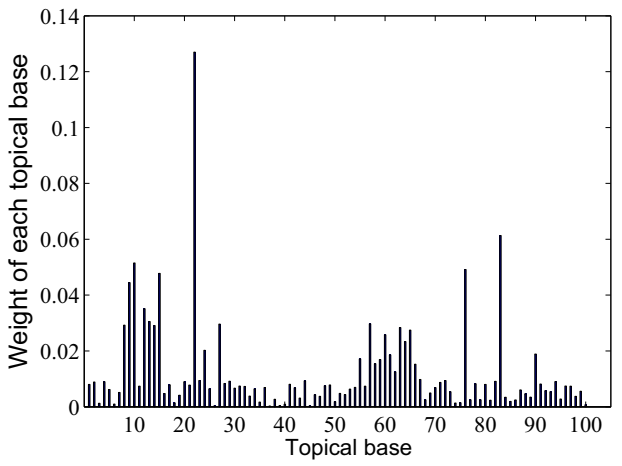

(b) Average document code of street

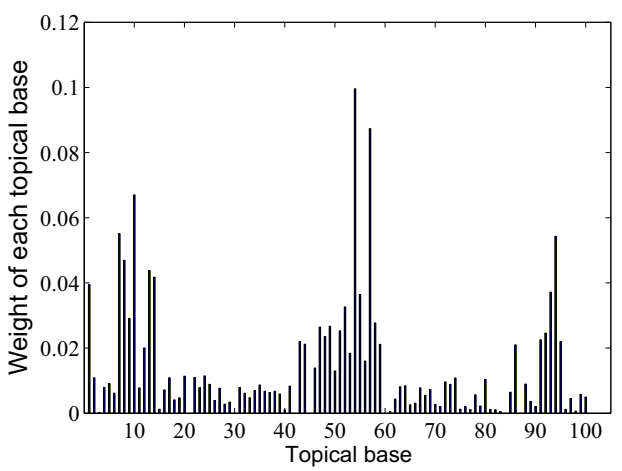

(d) Average document code of mountain

Figure 4: Average multi-modal document $\operatorname{code} \theta$ of four example categories in the LabelMe dataset. 
Multi-modal document code: Fig. 4 shows the average multi-modal document codes of four example categories in the LabelMe dataset when $K$ is fixed at 100. Regarding to each category, the average code is computed over all the multi-modal documents in that category and then is normalized. Apparently, the average multi-modal document codes are quite different with respect to different categories. Therefore, the codes learned by SMMTC have the power to distinguish different categories. Note that we can achieve the sparse code of each multi-modal document by imposing $l_{1}$-norm on $\theta$, whereas the average multi-modal document code is not sparse.

Image word code: Fig. 5 and Fig. 6 show average codes of some image words in four randomly selected images from the LabelMe and the Corel datasets when $K$ is fixed at 100, respectively. Average code weights $\bar{s}_{n k}$ of the image word $n$ is computed over all the multi-modal documents (indexed by $\mathscr{D}_{n}$ ) in which the image word appears, i.e., $\bar{s}_{n k}=\left(1 /\left|\mathscr{D}_{n}\right|\right) \sum_{d \in \mathscr{D}_{n}} s_{n k}^{d}$. As different images usually contain a varying number of image words, we just display the averaged codes of five image words that most frequently appear in each image. As seen from the figures, each averaged image word code has a few elements which are larger than others. It indicates that our model can control the sparsity of each image word code effectively.

\subsection{Automatic image annotation}

We first compare the performance of image annotation with the baselines on two benchmark datasets. Then, we perform sensitivity analyses of some hyperparameters to demonstrate the effectiveness of SMMTC on controlling the sparsity of inferred representations and predicting caption words for unannotated images. 

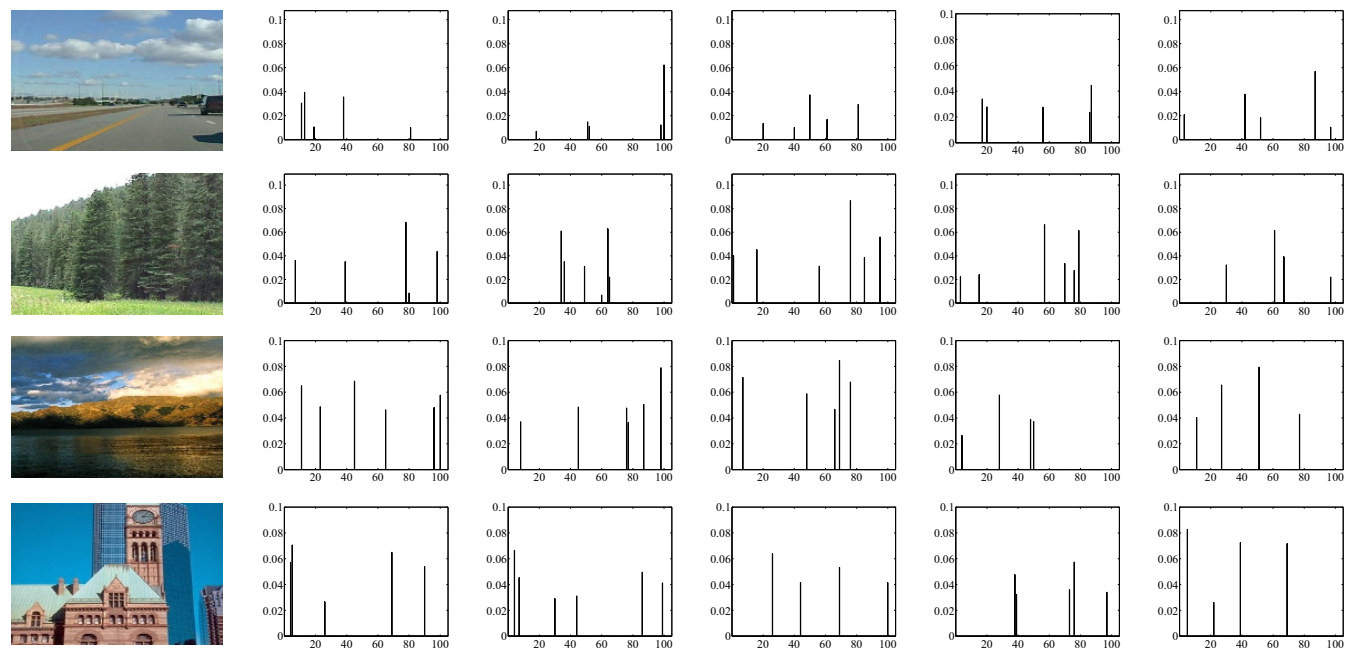

Figure 5: Average codes of five image words that most frequently appear in four images from the LabelMe dataset.
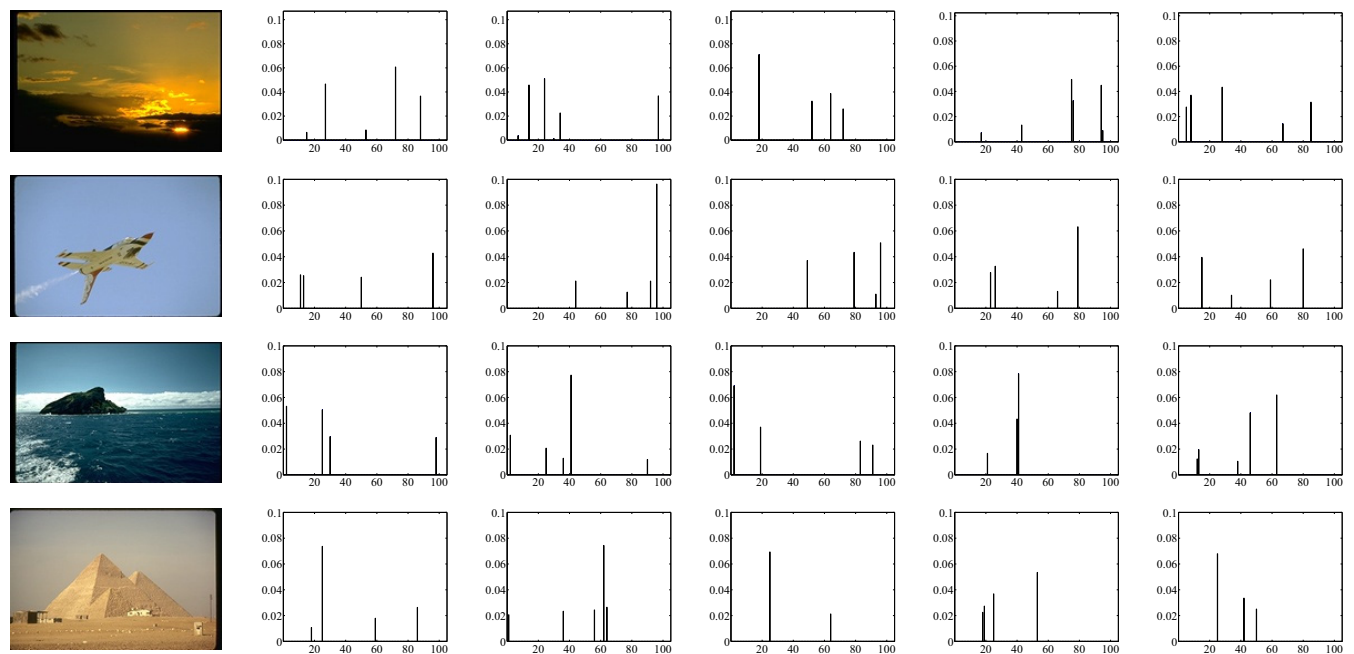

Figure 6: Average codes of five image words that most frequently appear in four images from the Corel dataset. 


\subsubsection{Caption perplexity}

We follow the baseline models $[11,12]$ and use the value of perplexity [40] to measure the performance of image annotation. The perplexity is defined as

$$
\text { perplexity }=\exp \left\{-\sum_{d=1}^{D^{\prime}} \sum_{n \in J^{d}} \log p\left(n \mid R_{d}\right) / \sum_{d=1}^{D^{\prime}}\left|J^{d}\right|\right\}
$$

where $D^{\prime}$ denotes the number of multi-modal documents in the test set and $p\left(n \mid R_{d}\right)$ represents the conditional probability of the caption word $n$ given the $d$-th test image $R_{d}$. Perplexity can be interpreted as the inverse of the geometric mean likelihood. Thus a lower perplexity means better prediction performance.

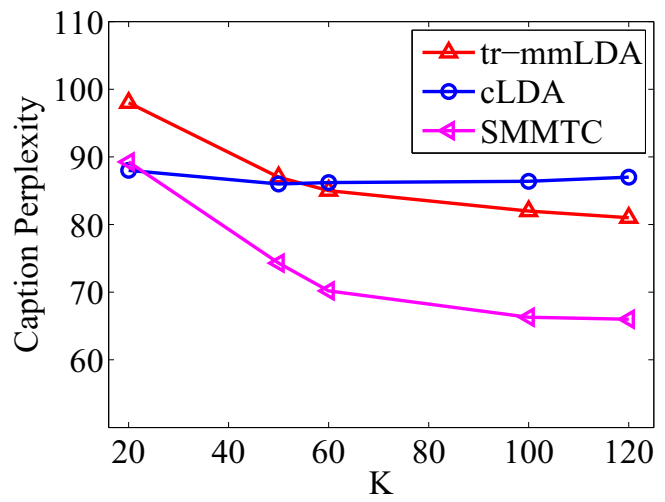

(a) Corel dataset

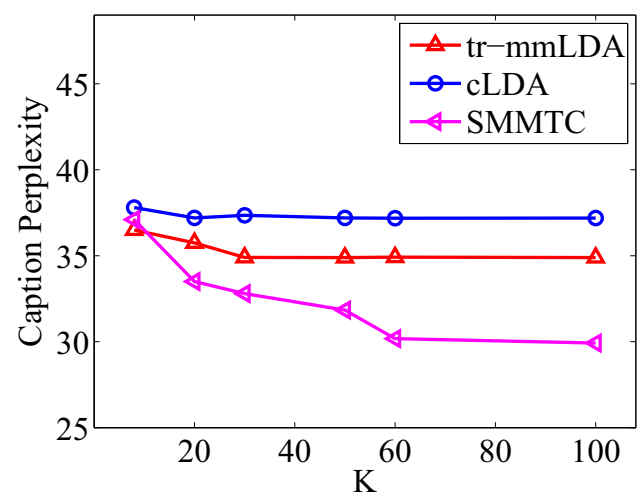

(b) LabelMe dataset

Figure 7: Caption perplexity with different values of $\mathrm{K}$ on different datasets.

By using the method applied in [54], we compute the conditional probability $p\left(n \mid R_{d}\right)$ via using the cosine similarity between the image code and the caption word code. The image code $\mathbf{s}^{d}$ of $R_{d}$ is computed by combining the average codes of image words that appear in this image, i.e., $\mathbf{s}^{d}=\sum_{m \in I^{d}} r_{m}^{d} \overline{\mathbf{s}}_{m}^{d}$. The word code $\overline{\mathbf{z}}_{n}$ of $n$ is computed over all the training multi-modal documents (indexed by $C_{n}$ ) in which $n$ appears, i.e., $\overline{\mathbf{z}}_{n}=\left(1 /\left|C_{n}\right|\right) \sum_{d \in C_{n}} \mathbf{z}_{n}^{d}$. 
Fig. 7 shows the caption perplexities of each model with different values of $K$. We can see that SMMTC achieves a lower perplexity value than the baseline models. In contrast, cLDA gets the worst performance which can be attributed to its severe restriction on the relationships between image regions and caption words. We show in Fig. 8 six example images from the test set of LabelMe and their annotations predicted by each model when $K=100$. The incorrect predictions appear in red font.
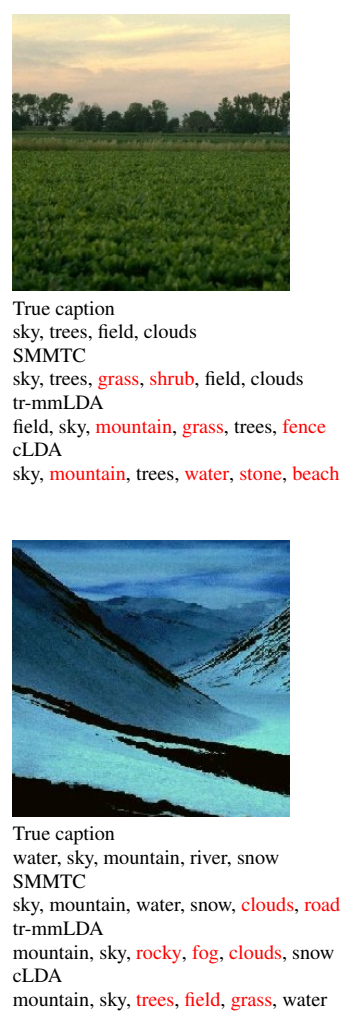
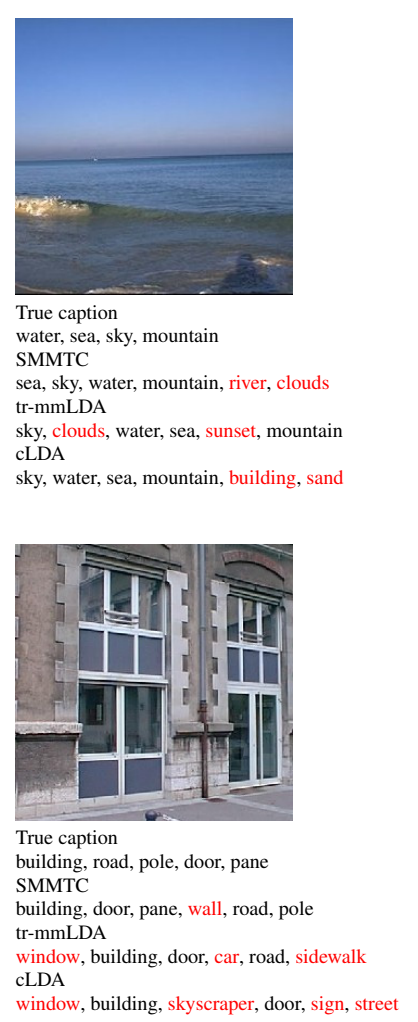

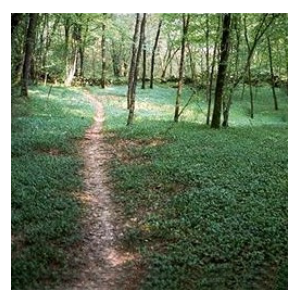

True caption

trees, trunk, ground, grass, path SMMTC

trees, grass, path, trunk, shrub, ground tr-mmLDA

trees, trunk, sky, forest, ground, slope cLDA

trees, trunk, sky, mountain, grass, pillar

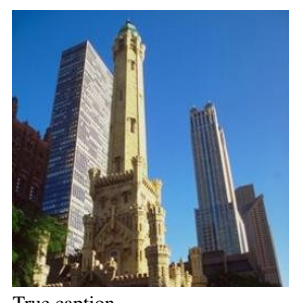

True caption

sky, building, trees, skyscraper

作, sky, tower, building, trees, clouds tr-mmLDA

building, skyscraper, sky, road, rock, car

cLDA
building, skyscraper, sky, trees, street, water

Figure 8: Example images from the test set of LabelMe and their annotations predicted by different models.

SMMTC can achieve better performance on image annotation for two reasons: i) SMMTC can effectively learn sparse image representations, which are mean- 
ingful in image understanding and conform with the mechanism of mammalian primary visual cortex. ii) Compared with baseline models, SMMTC can capture more compact correlations between image regions and caption words. SMMTC encourages each caption word to be associated with a few image regions. It means that our model is more applicable to scenarios in which a word only describes partial content of an image. In contrast, cLDA restricts each caption word to be associated with a particular image region, which doesn't consider the fact that some words describe more than one image regions. Tr-mmLDA tends to correlate a word with all the image regions indistinguishably.

\subsubsection{Sensitivity analysis on hyper-parameters}

The advantage of SMMTC is reflected in its flexibility to control the sparsity of image word codes and correlation codes by regulating the hyper-parameters $(\rho, \lambda, \gamma, \mu)$. In this section, we present the sparsity ratios of image word codes and correlation codes with respect to different numbers of topical bases, where the sparsity ratio is defined as the average ratio of zero elements in codes. Besides, we analyze how the hyper-parameters affect caption perplexity. Recalling optimization problem (4), the sparsity of image word codes and correlation codes can be regulated by adjusting the parameters $\rho$ and $\mu$, respectively. Following $[17,42]$, we fix $\lambda=\gamma$ and only tune the ratios $\rho / \gamma$ and $\mu / \gamma$.

We fix $\mu$ and tune the ratio $\rho / \gamma$ to observe the variation of the sparsity ratio of image word codes over the Corel dataset. The results are shown in Fig. 9 (a). Correspondingly, we display the variations of caption perplexity when tuning the $\rho / \gamma$ in Fig. 9 (c). After fixing $\rho$, we show in Fig. 9 (b) and Fig. 9 (d) how the sparsity ratios of correlation codes and the corresponding caption perplexity vary when tuning the ratio $\mu / \gamma$ over the Corel dataset. We also perform the same 


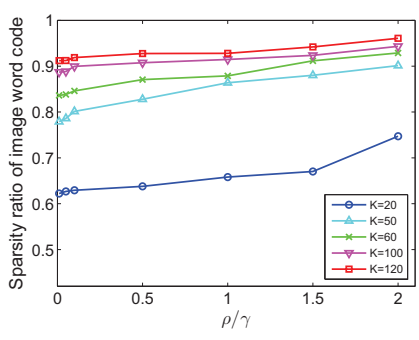

(a) Sparsity ratios of image word codes when tuning the ratio $\rho / \gamma$.

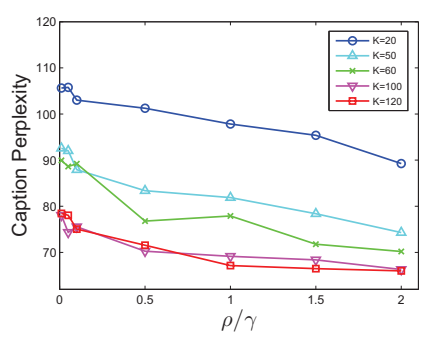

(c) Caption perplexities when tuning the ratio $\rho / \gamma$.

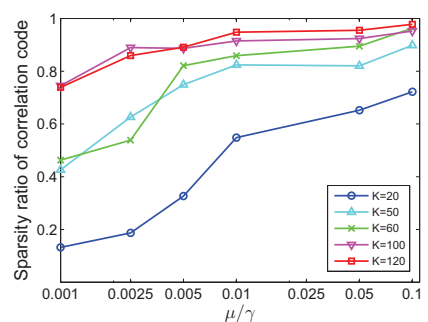

(b) Sparsity ratios of correlation codes when tuning the ratio $\mu / \gamma$.

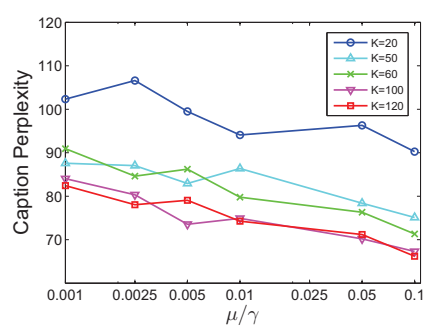

(d) Caption perplexities when tuning the ratio $\mu / \gamma$.

Figure 9: Sparsity ratios and caption perplexities with different values of K over the Corel dataset.

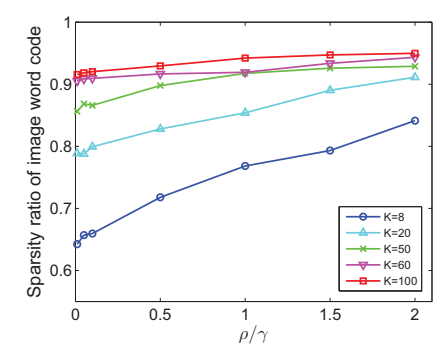

(a) Sparsity ratios of image word codes when tuning the ratio $\rho / \gamma$.

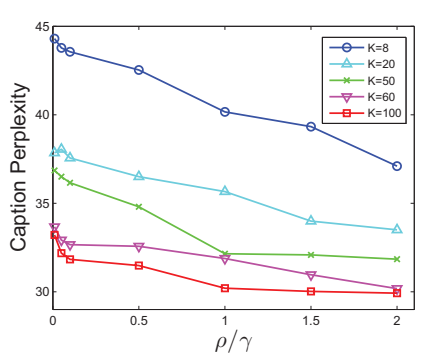

(c) Caption perplexities when tuning the ratio $\rho / \gamma$.

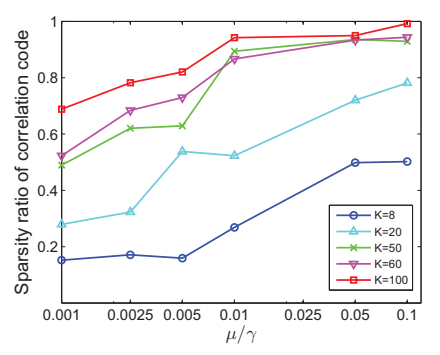

(b) Sparsity ratios of correlation codes when tuning the ratio $\mu / \gamma$.

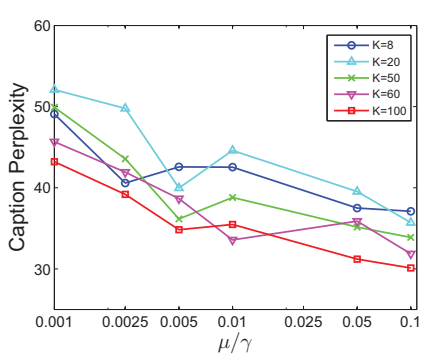

(d) Caption perplexities when tuning the ratio $\mu / \gamma$.

Figure 10: Sparsity ratios and caption perplexities with different values of K over the LabelMe dataset. 
sensitivity analyses over the LabelMe dataset and show the results in Fig. 10.

From the figures, we can draw the following two observations: i) When the number of topical bases is relatively small, increasing $\rho$ and $\mu$ can gradually improve the sparsity ratios of image word codes and correlation codes, respectively. It is obvious that the results consist with the formulation in the optimization problem (4). ii) Gradual reduction in caption perplexity can be seen, when $\rho$ and $\mu$ grows, respectively. It indicates that, to some extend, sparser representations of images and correlations between images and words can lead to better annotation performance. The reason for the reduction is that SMMTC captures more compact correlations between image regions and words via improving the sparsity of correlation codes.

\subsection{Text-based image retrieval}

Following the method in baseline models $[11,12]$, we use SMMTC for textbased image retrieval. Given a single word query $q$, image retrieval is performed by ranking images according to their relativity to $q$. For a test image $R_{t e s t}$, its relative score to $q$ is computed by score $_{R_{\text {test }}}=p\left(q \mid R_{\text {test }}\right)$, where $p\left(q \mid R_{\text {test }}\right)$ denotes the probability of the image $R_{\text {test }}$ annotated with the query word $q$. The probability is computed in 4.3 .

With respect to three single word queries, Fig. 11 depicts the precision-recall curves produced by each model when $K$ is fixed at 100. It shows that SMMTC achieves higher precision at the same recall values for each query. Besides, in order to describe the results of image retrieval more intuitively, we show in Fig. 12 top six images retrieved from the LabelMe dataset with query words "grass," "river" and "house," respectively. The irrelevant images are highlighted by red boxes. For the query word "river," two irrelevant images appear in the returned 


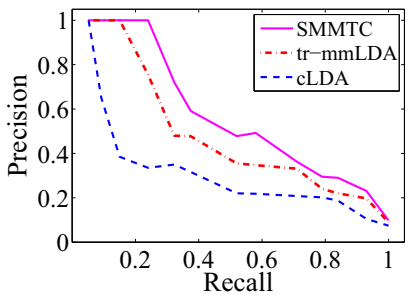

(a) wave

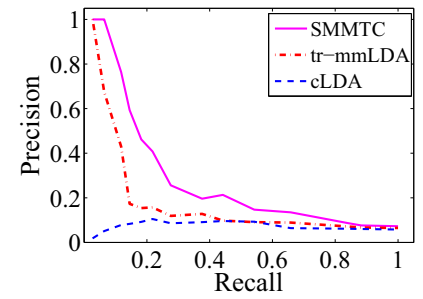

(b) pole

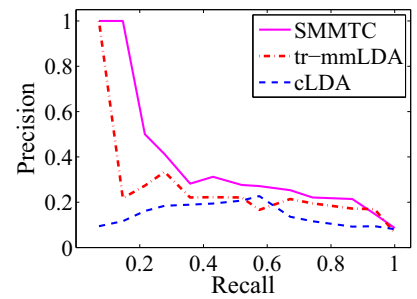

(c) flowers

Figure 11: Precision-recall curves for three word queries on the LabelMe dataset using different models.

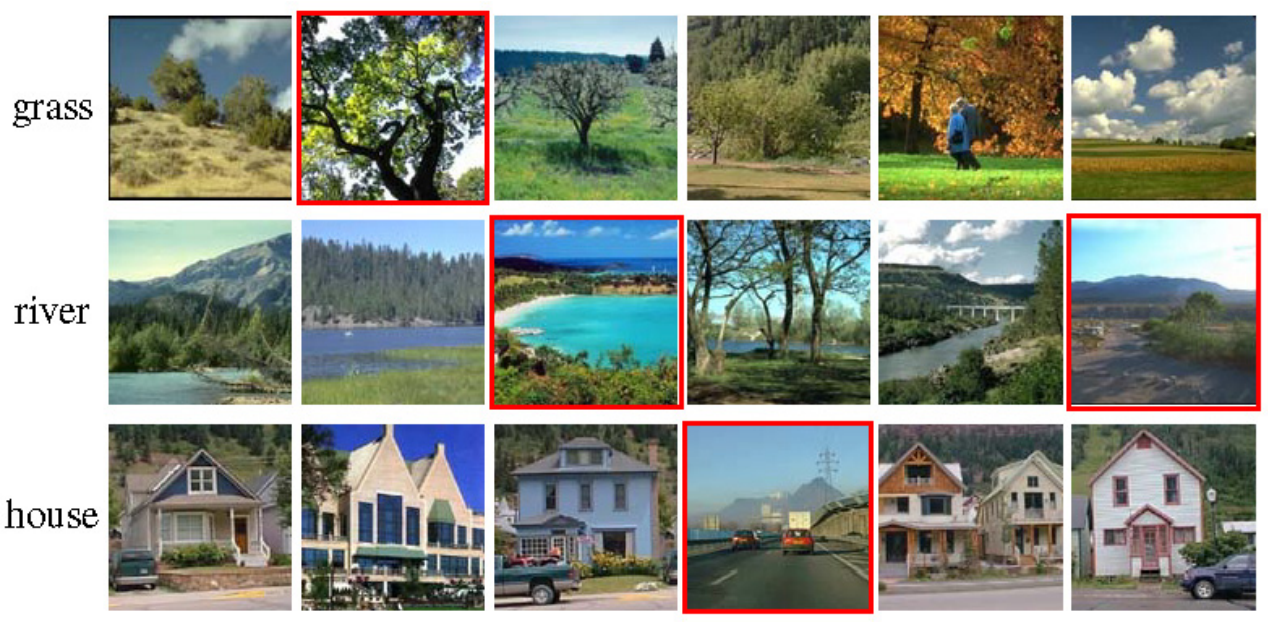

Figure 12: Top six images retrieved using single word queries over the LabelMe dataset.

results. As to query words "grass" and "house," there are five relevant images appearing in the top six retrieval results, respectively.

\section{Conclusions and future work}

In this work, we have proposed SMMTC, a non-probabilistic formulation of PTM for image annotation. Without the normalization constraints of probabilistic topic models, SMMTC can effectively control the sparsity of inferred latent representations. Besides, it depicts a more accurate relationship for caption words that 
only describe a few image regions. Experimental results over some benchmark datasets demonstrate that SMMTC outperforms the baseline models for the task of image annotation and retrieval.

For future work, we are interested in exploring relations between different multi-modal documents and utilizing such relations to develop relational SMMTC for automatic image annotation in social media.

\section{Acknowledgments}

The research was supported in part by the National Science Foundation of China under Grant Nos. 61502377, 61532015, 61532004; Ministry of Education Innovation Research Team under Grant No. IRT13035.

\section{References}

[1] M. Srinivas, R. R. Naidu, C. Sastry, C. K. Mohan, Content based medical image retrieval using dictionary learning, Neurocomputing 168 (2015) 880895.

[2] D. Feng, J. Yang, C. Liu, An efficient indexing method for content-based image retrieval, Neurocomputing 106 (2013) 103-114.

[3] W. Li, L. Duan, D. Xu, I. W.-H. Tsang, Text-based image retrieval using progressive multi-instance learning, in: IEEE International Conference on Computer Vision, 2011, pp. 2049-2055.

[4] L. Wu, R. Jin, A. K. Jain, Tag completion for image retrieval, IEEE Transactions on Pattern Analysis and Machine Intelligence 35 (3) (2013) $716-727$. 
[5] G. Carneiro, N. Vasconcelos, Formulating semantic image annotation as a supervised learning problem, in: Proceedings of the IEEE Computer Society Conference on Computer Vision and Pattern Recognition, Vol. 2, 2005, pp. $163-168$.

[6] J. Li, J. Z. Wang, Automatic linguistic indexing of pictures by a statistical modeling approach, IEEE Transactions on Pattern Analysis and Machine Intelligence 25 (9) (2003) 1075-1088.

[7] Z. Qian, P. Zhong, J. Chen, Integrating global and local visual features with semantic hierarchies for two-level image annotation, Neurocomputing 171 (2016) 1167-1174.

[8] R. Hong, M. Wang, Y. Gao, D. Tao, X. Li, X. Wu, Image annotation by multiple-instance learning with discriminative feature mapping and selection, IEEE Transactions on Cybernetics 44 (5) (2014) 669-680.

[9] C. Xu, D. Tao, C. Xu, A survey on multi-view learning, arXiv preprint arXiv:1304.5634.

[10] X. Chang, F. Nie, Y. Yang, H. Huang, A convex formulation for semisupervised multi-label feature selection, in: AAAI, 2014, pp. 1171-1177.

[11] D. M. Blei, M. I. Jordan, Modeling annotated data, in: Proceedings of the 26th international ACM SIGIR conference on Research and development in informaion retrieval, 2003, pp. 127-134.

[12] D. Putthividhy, H. T. Attias, S. S. Nagarajan, Topic regression multi-modal latent dirichlet allocation for image annotation, in: Proceedings of the IEEE 
Conference on Computer Vision and Pattern Recognition, 2010, pp. 34083415.

[13] K. Barnard, P. Duygulu, D. Forsyth, N. De Freitas, D. M. Blei, M. I. Jordan, Matching words and pictures, Journal of Machine Learning Research 3 (2003) 1107-1135.

[14] O. Yakhnenko, V. Honavar, Annotating images and image objects using a hierarchical dirichlet process model, in: Proceedings of the 9th International Workshop on Multimedia Data Mining, 2008, pp. 1-7.

[15] C. Wang, D. Blei, F.-F. Li, Simultaneous image classification and annotation, in: Proceedings of the IEEE Conference on Computer Vision and Pattern Recognition, 2009, pp. 1903-1910.

[16] K. Ganchev, B. Taskar, F. Pereira, J. Gama, Posterior vs parameter sparsity in latent variable models, in: Proceedings of Advances in Neural Information Processing Systems, 2009, pp. 664-672.

[17] J. Zhu, E. Xing, Sparse topical coding, in: Proceedings of the TwentySeventh Conference Annual Conference on Uncertainty in Artificial Intelligence, 2011, pp. 831-838.

[18] J. Wright, Y. Ma, J. Mairal, G. Sapiro, T. S. Huang, S. Yan, Sparse representation for computer vision and pattern recognition, Proceedings of the IEEE 98 (6) (2010) 1031-1044.

[19] W. Zhao, Z. Liu, Z. Guan, B. Lin, D. Cai, Orthogonal projective sparse coding for image representation, Neurocomputing 173 (2016) 270-277. 
[20] L. Zhang, C. Ma, Low-rank decomposition and laplacian group sparse coding for image classification, Neurocomputing 135 (2014) 339-347.

[21] K. Cheng, X. Tan, Sparse representations based attribute learning for flower classification, Neurocomputing 145 (2014) 416-426.

[22] C. Shi, Q. Ruan, S. Guo, Y. Tian, Sparse feature selection based on $l_{2,1 / 2^{-}}$ matrix norm for web image annotation, Neurocomputing 151 (2015) 424433.

[23] W. Liu, D. Tao, J. Cheng, Y. Tang, Multiview hessian discriminative sparse coding for image annotation, Computer Vision and Image Understanding 118 (2014) 50-60.

[24] H. Du, X. Zhang, Q. Hu, Y. Hou, Sparse representation-based robust face recognition by graph regularized low-rank sparse representation recovery, Neurocomputing 164 (2015) 220-229.

[25] H. Liu, Y. Liu, F. Sun, Robust exemplar extraction using structured sparse coding, IEEE Transactions on Neural Networks and Learning Systems 26 (8) (2015) 1816-1821.

[26] G. Carneiro, A. B. Chan, P. J. Moreno, N. Vasconcelos, Supervised learning of semantic classes for image annotation and retrieval, IEEE Transactions on Pattern Analysis and Machine Intelligence 29 (3) (2007) 394-410.

[27] D. Tao, L. Jin, W. Liu, X. Li, Hessian regularized support vector machines for mobile image annotation on the cloud, IEEE Transactions on Multimedia 15 (4) (2013) 833-844. 
[28] C. Xu, T. Liu, D. Tao, C. Xu, Local rademacher complexity for multi-label learning, IEEE Transactions on Image Processing 25 (3) (2016) 1495-1507.

[29] R. S. Cabral, F. De la Torre, J. P. Costeira, A. Bernardino, Matrix completion for multi-label image classification, in: Proceedings of Advances in Neural Information Processing Systems, Vol. 201, 2011, pp. 190-198.

[30] Y. Luo, D. Tao, B. Geng, C. Xu, S. J. Maybank, Manifold regularized multitask learning for semi-supervised multilabel image classification, IEEE Transactions on Image Processing 22 (2) (2013) 523-536.

[31] Z. Wei, H. Wang, R. Zhao, Semi-supervised multi-label image classification based on nearest neighbor editing, Neurocomputing 119 (2013) 462-468.

[32] C. Xu, D. Tao, C. Xu, Multi-view intact space learning, IEEE Transactions on Pattern Analysis and Machine Intelligence 37 (12) (2015) 2531-2544.

[33] C. Xu, D. Tao, C. Xu, Large-margin multi-view information bottleneck, IEEE Transactions on Pattern Analysis and Machine Intelligence 36 (8) (2014) 1559-1572.

[34] W. Liu, D. Tao, Multiview hessian regularization for image annotation, IEEE Transactions on Image Processing 22 (7) (2013) 2676-2687.

[35] Y. Luo, T. Liu, D. Tao, C. Xu, Multiview matrix completion for multilabel image classification, IEEE Transactions on Image Processing 24 (8) (2015) $2355-2368$.

[36] Y. Luo, D. Tao, C. Xu, C. Xu, H. Liu, Y. Wen, Multiview vectorvalued manifold regularization for multilabel image classification, IEEE 
Transactions on Neural Networks and Learning Systems 24 (5) (2013) 709722.

[37] J. Jeon, V. Lavrenko, R. Manmatha, Automatic image annotation and retrieval using cross-media relevance models, in: Proceedings of the 26th annual international ACM SIGIR conference on Research and development in informaion retrieval, 2003, pp. 119-126.

[38] V. Lavrenko, R. Manmatha, J. Jeon, A model for learning the semantics of pictures, Advances in neural information processing systems (2003) 553560.

[39] C. Wang, F. Jing, L. Zhang, H.-J. Zhang, Content-based image annotation refinement, in: IEEE Conference on Computer Vision and Pattern Recognition, 2007, pp. 1-8.

[40] D. M. Blei, A. Y. Ng, M. I. Jordan, Latent dirichlet allocation, Journal of machine Learning research 3 (2003) 993-1022.

[41] C. Wang, D. M. Blei, Decoupling sparsity and smoothness in the discrete hierarchical dirichlet process, in: Advances in neural information processing systems, 2009, pp. 1982-1989.

[42] A. Zhang, J. Zhu, B. Zhang, Sparse relational topic models for document networks, in: Proceedings of European Conference on Machine Learning and Principles and Practice of Knowledge Discovery in Databases, 2013, pp. 670-685.

[43] R. Tibshirani, Regression shrinkage and selection via the lasso, Journal of the Royal Statistical Society. Series B (1996) 267-288. 
[44] D. D. Lee, H. S. Seung, Learning the parts of objects by non-negative matrix factorization, Nature 401 (6755) (1999) 788-791.

[45] W. D. Blizard, Multiset theory, Notre Dame Journal of formal logic 30 (1) (1989) 36-66.

[46] A. Hyvärinen, Sparse code shrinkage: Denoising of nongaussian data by maximum likelihood estimation, Neural computation 11 (7) (1999) 17391768.

[47] A. Zhang, J. Zhu, B. Zhang, Sparse online topic models, in: Proceedings of the 22nd international conference on World Wide Web, 2013, pp. 14891500 .

[48] J. Duchi, S. Shalev-Shwartz, Y. Singer, T. Chandra, Efficient projections onto the $l_{1}$-ball for learning in high dimensions, in: Proceedings of the 25 th international conference on Machine learning, 2008, pp. 272-279.

[49] B. C. Russell, A. Torralba, K. P. Murphy, W. T. Freeman, Labelme: a database and web-based tool for image annotation, International journal of computer vision 77 (1-3) (2008) 157-173.

[50] P. Duygulu, K. Barnard, J. F. de Freitas, D. A. Forsyth, Object recognition as machine translation: Learning a lexicon for a fixed image vocabulary, in: Proceedings of the European conference on computer vision, 2002, pp. 97-112.

[51] J. C. Caicedo, J. BenAbdallah, F. A. González, O. Nasraoui, Multimodal representation, indexing, automated annotation and retrieval of image collec- 
tions via non-negative matrix factorization, Neurocomputing 76 (1) (2012) 50-60.

[52] M. Wang, F. Li, M. Wang, Collaborative visual modeling for automatic image annotation via sparse model coding, Neurocomputing 95 (2012) 2228.

[53] J. V. D. Weijer, C. Schmid, Coloring local feature extraction, in: Proceedings of the 9th European Conference on Computer Vision, Vol. 2, 2006, pp. 334348.

[54] N. Coccaro, D. Jurafsky, Towards better integration of semantic predictors in statistical language modeling, in: Proceedings of the 5th International Conference on Spoken Language Processing, 1998, pp. 2403-2406. 
Lingyun Song received the M.S. degree in software engineering from Xi'an Jiaotong University, China, in 2014. Currently, he is working toward the Ph.D. degree in computer science at Xi'an Jiaotong University, China. His research interests include machine learning, pattern recognition, image understanding and searching.

Minnan Luo received her Ph. D. degree from the Department of Computer Science and Technology, Tsinghua University, Beijing, China, in 2014. Currently, she is an Assistant Professor in the School of Electronic and Information Engineering at Xi'an Jiaotong University. She is also a Post-Doctoral Researcher with the School of Computer Science, Carnegie Mellon University, PA, USA. Her research interests include machine learning and optimization, video analysis, cross-media retrieval and fuzzy system modeling.

Jun Liu received the B.S., M.S., and Ph.D. degrees from Xi'an Jiaotong University, China, in 1995, 1998, and 2004, respectively, all in computer science. He is currently a professor in the Department of Computer Science, Xi'an Jiaotong University. His main research interests include text mining, data mining and e-learning. He has published more than 40 research papers in various journals and conference proceedings.

Lingling Zhang received her B.S. degree in computer science from Xi'an Jiaotong University, China, in 2014. Currently, she is working toward the M.S. degree in computer science at Xi'an Jiaotong University, China. Her research interests include data mining, pattern recognition, image understanding and searching.

Buyue Qian is currently an associate professor at Xi'An Jiaotong University. Before that he worked as a research scientist at IBM T. J. Watson Research. He received his PhD in 2013 from Computer Science Department, University of California at Davis. Before that, he received Master of Science (2009) from Columbia University, and BS in Information Engineering (2007) from Xi'an Jiaotong University. The major awards he received include Yahoo! Research Award, IBM Eminence I\& Excellence Award, and SIAM Data Mining'13 Best Research Paper Runner Up.

Max Haifei Li is an associate professor of computer science at Union University, Jackson, TN. He worked for Nyack College before Union University. He was a post doctoral researcher at IBM Thomas J. Watson Reseach Center before Nyack College. He worked for China Resources Information Technology (CRIT) and China National Petroleum Corporation (CNPC) before coming to US for graduate degrees. His Master's and Ph.D. degrees are from the University of Florida and he got his Bachelor's degree from Xi'an Jiaotong University. He has published more than 30 papers. He is a senior member of both IEEE and ACM. He is the faculty advisor of the ACM student chapter at Union University.

Qinghua Zheng received the B.S. degree in computer software in 1990, the MS degree in computer organization and architecture in 1993, and the PhD degree in system engineering in 1997 from Xi'an Jiaotong University, China. He is currently a professor in Xi'an Jiaotong University, and the dean of the Department of Computer Science. His research areas include multimedia distance education, and computer network security. 


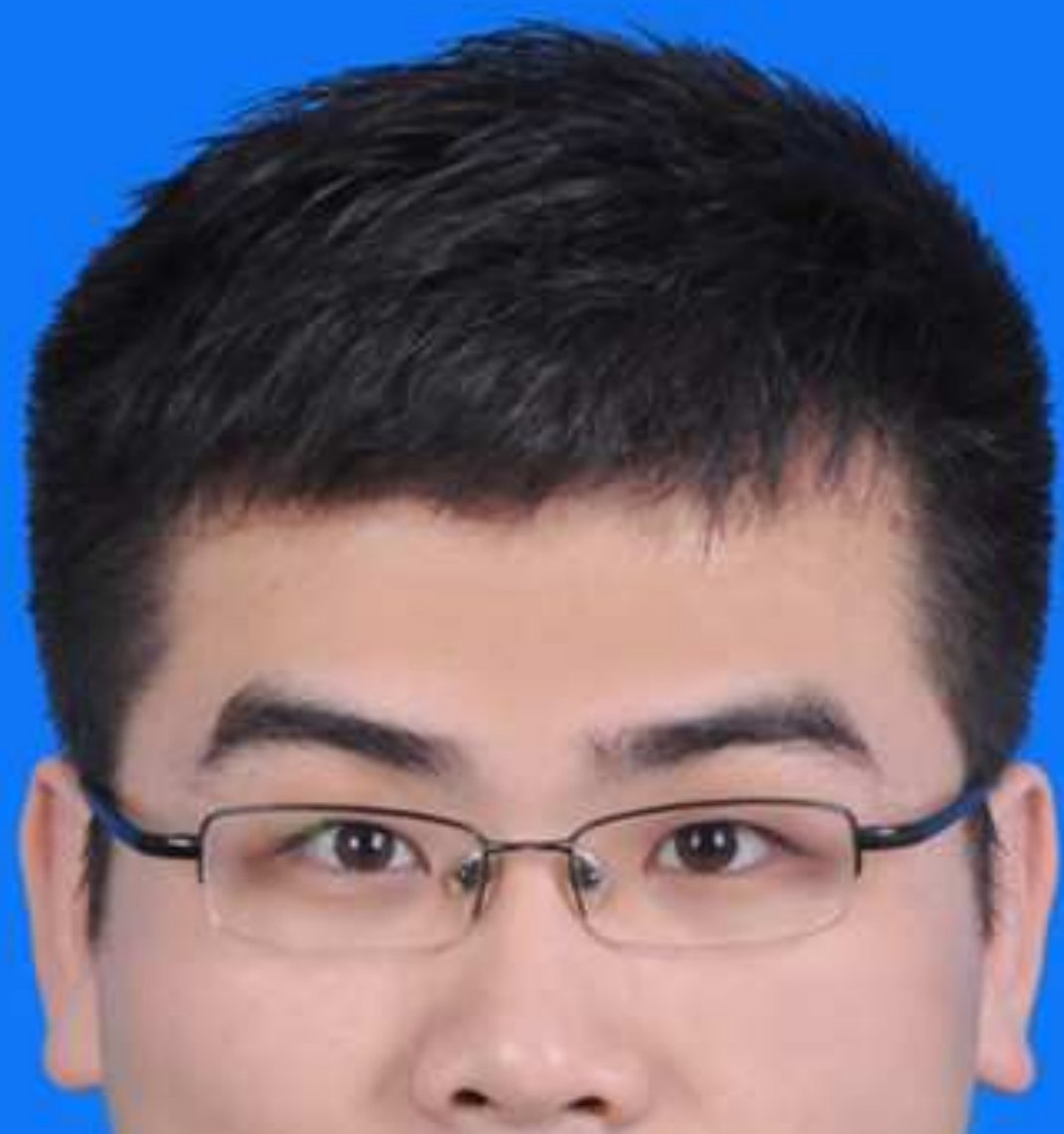




\section{${ }^{*}$ Photo of the author(s)}
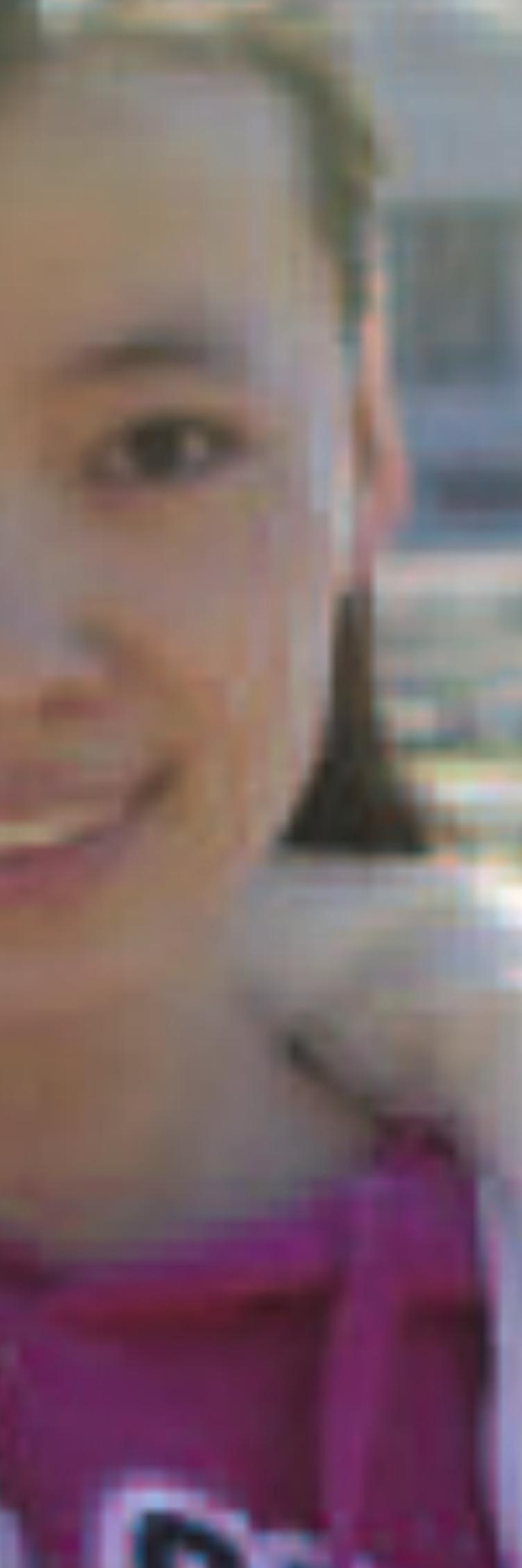

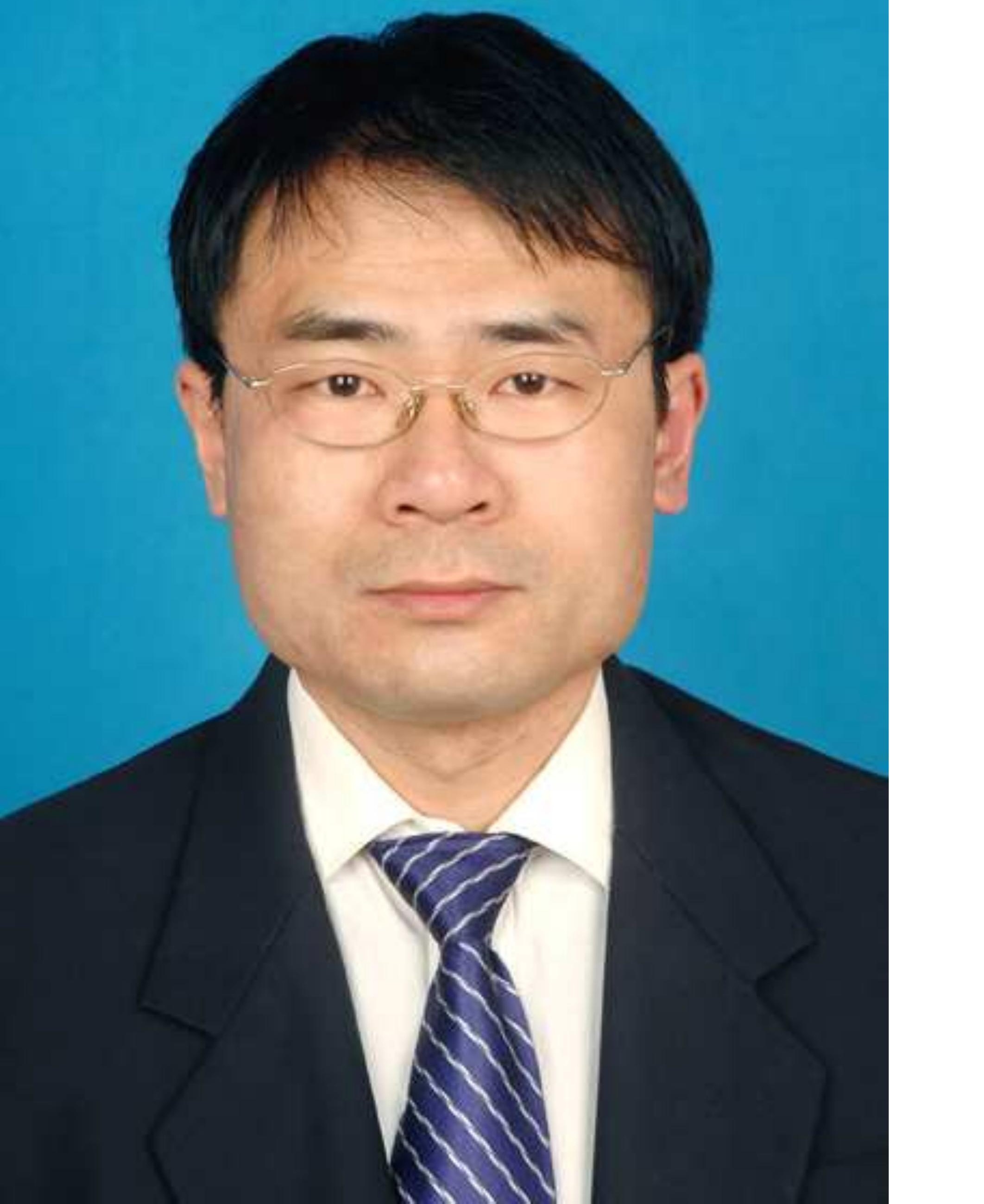

\section{${ }^{*}$ Photo of the author(s)}

s)

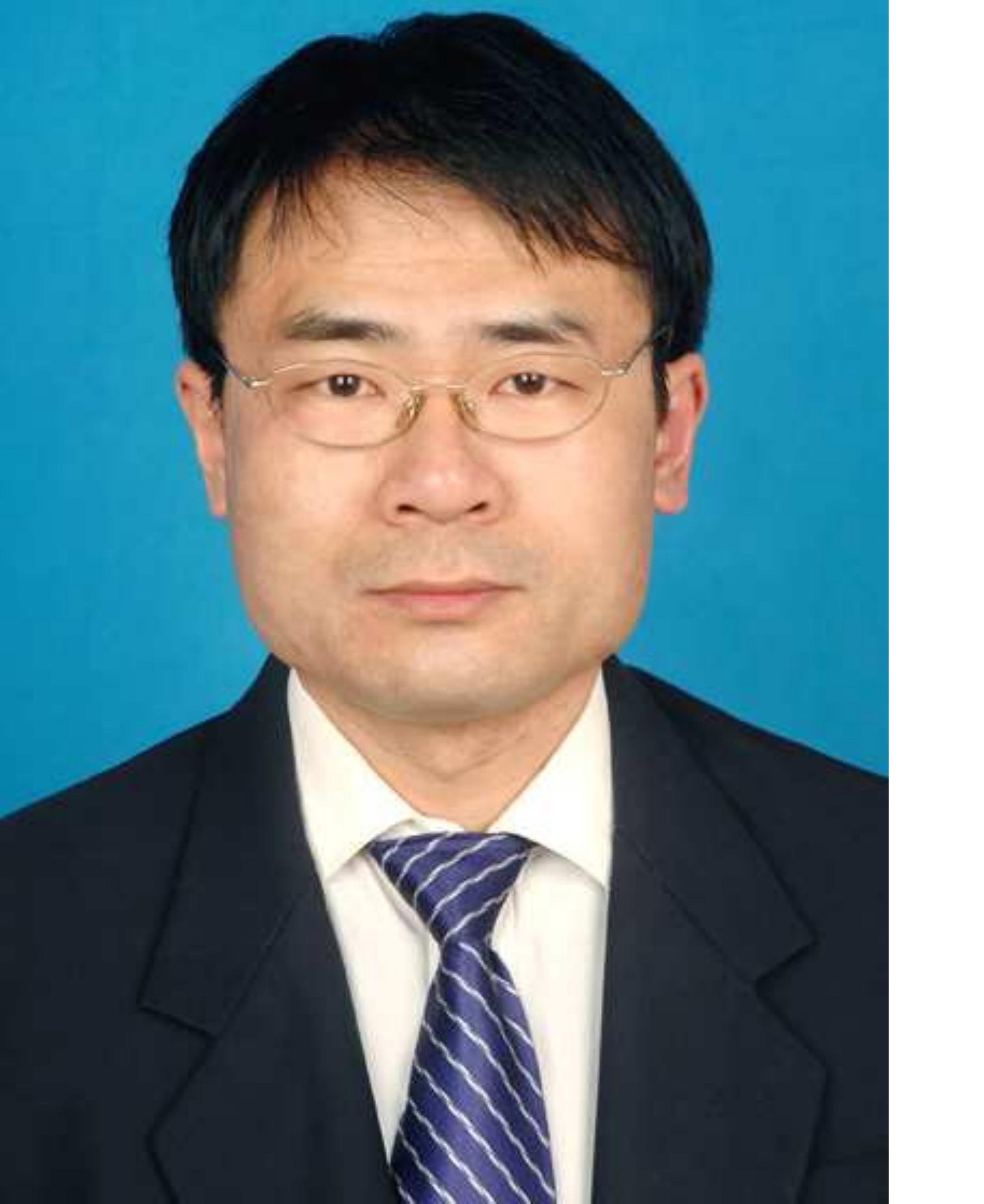




\section{${ }^{*}$ Photo of the author(s)}
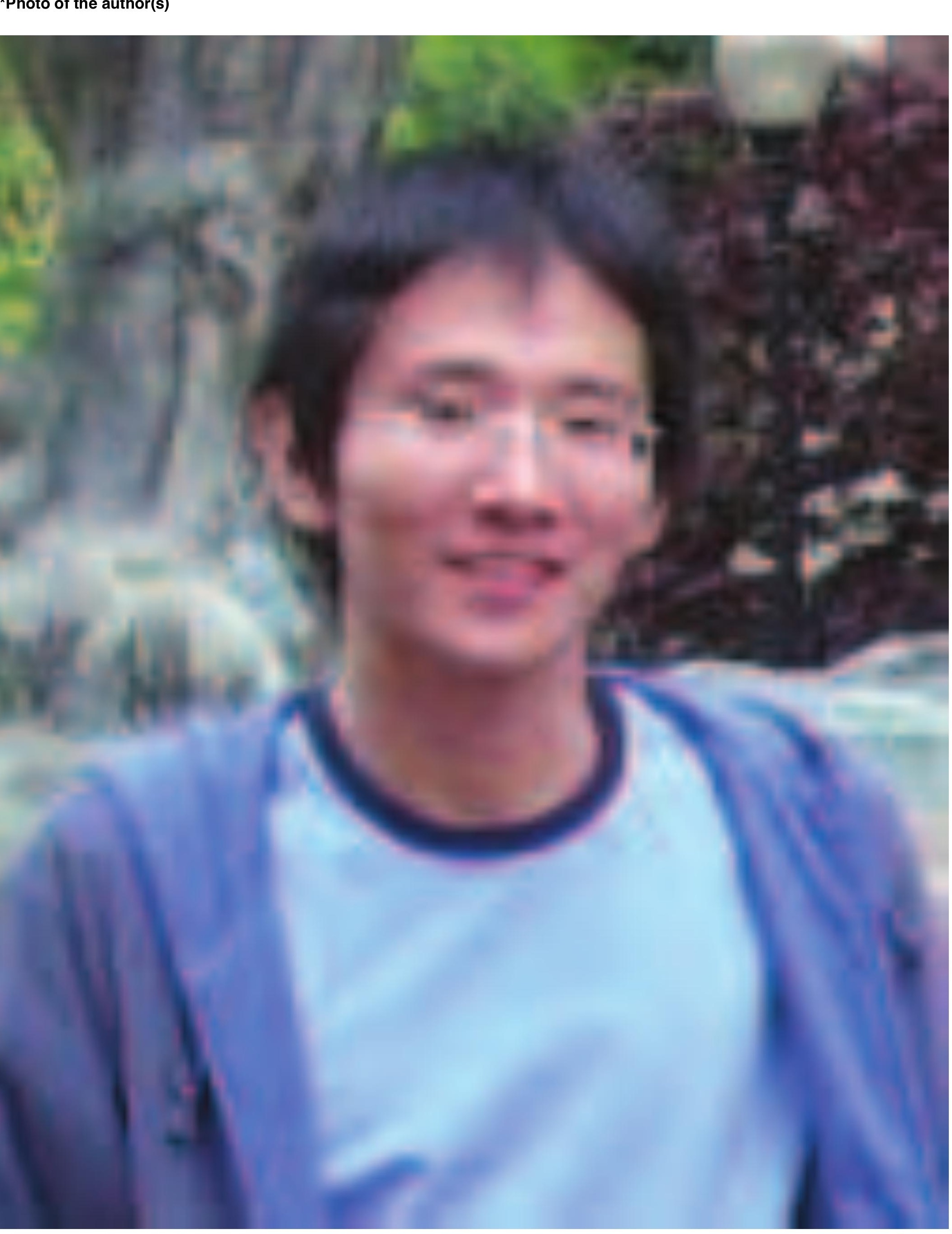
${ }^{*}$ Photo of the author(s)

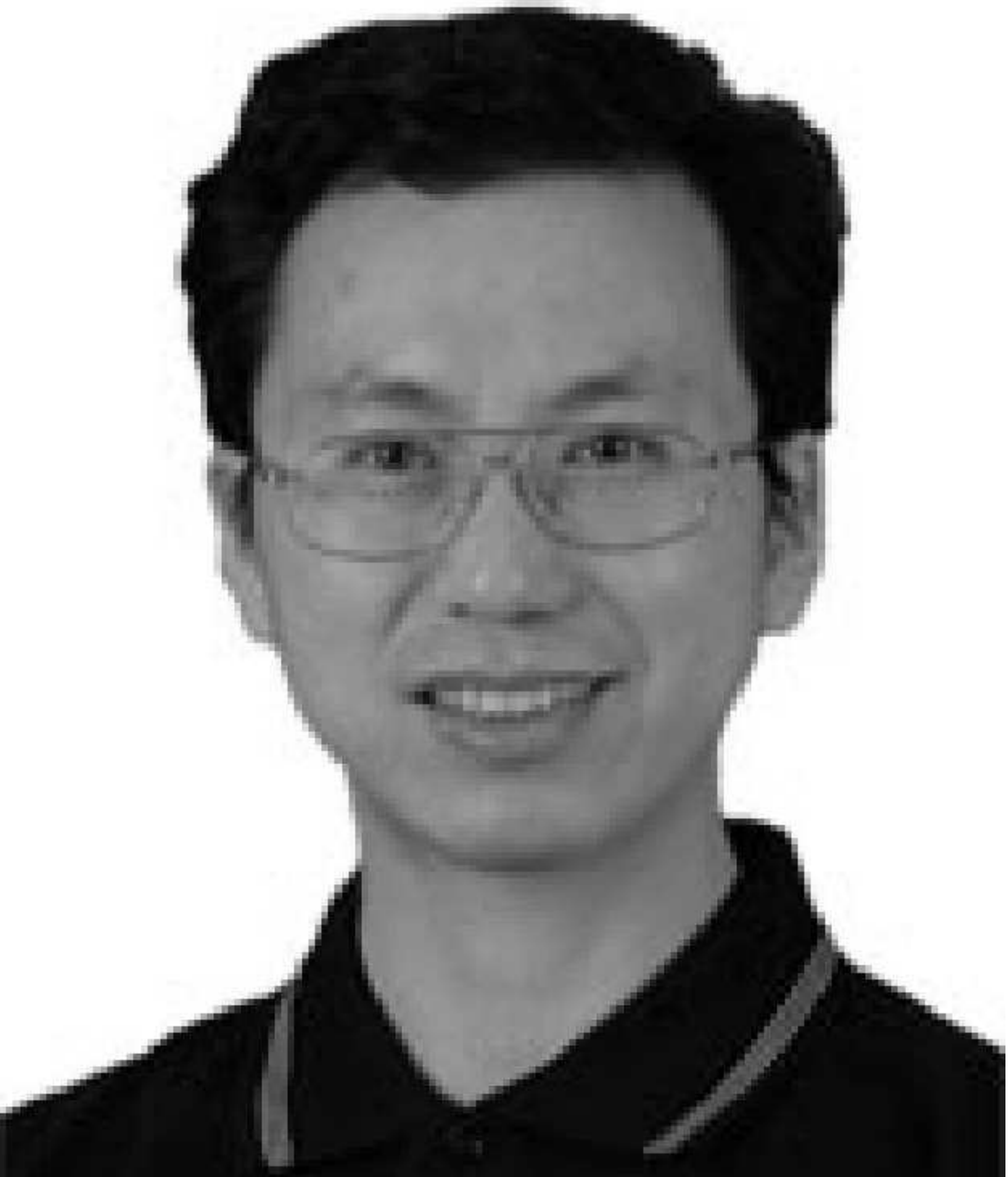


${ }^{*}$ Photo of the author(s)
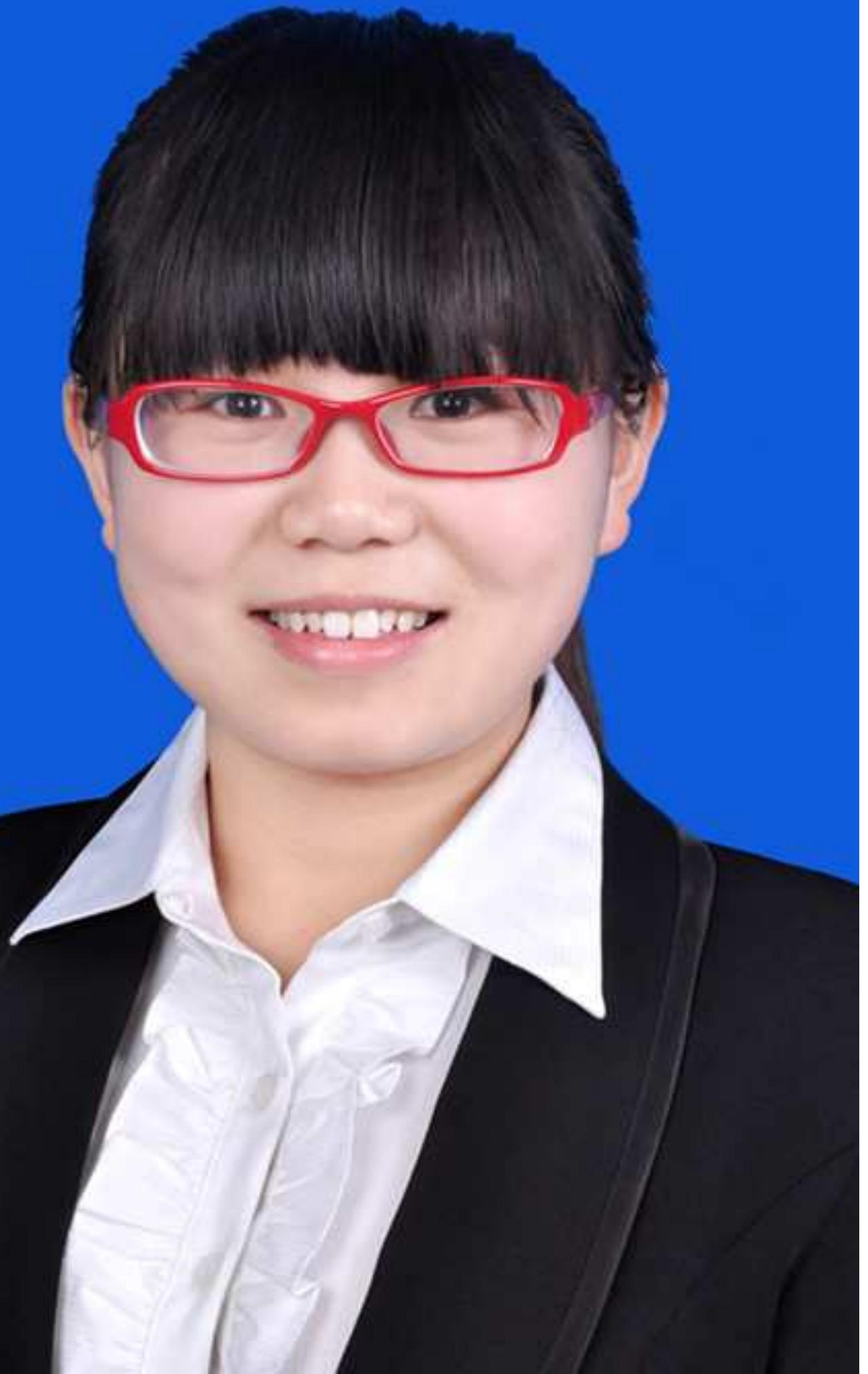
${ }^{*}$ Photo of the author(s)

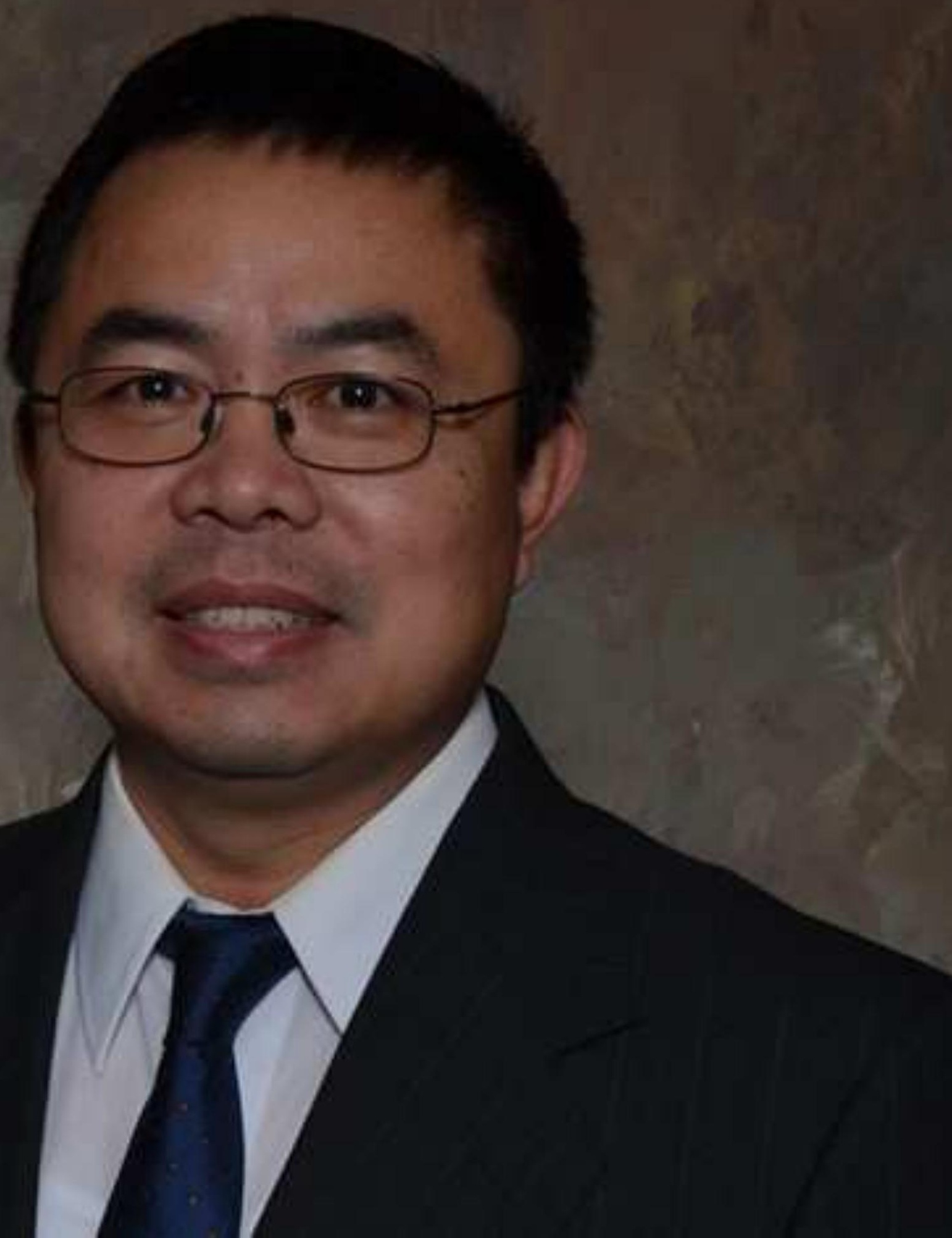

